\title{
On Properties of Fuzzy Mealy Machines
}

\author{
S. A. Morye \\ Department of Mathematics \\ Rajaram College, Kolhapur(M.S.)-416 004 \\ S. R. Chaudhari \\ Department of Mathematics \\ School of Mathematical Sciences, North Maharashtra University, Jalgaon (M.S.) - 425001
}

\begin{abstract}
It has been shown that the problem of equivalent and minimization of fuzzy Mealy machines can be resolved via their algebraic study. However, no attention has paid to study fuzzy Mealy machines topologically. This paper introduces topology on the state set of a fuzzy Mealy machine and study of various kinds of fuzzy Mealy machines viz. cyclic, retrievable, strongly connected, with exchange property and connected through this topology. In addition, various products of fuzzy Mealy machines and their relationship in regards to aforementioned kinds of fuzzy Mealy machines are also studied.
\end{abstract}

\section{Keywords:}

Fuzzy finite state machines, Fuzzy Mealy machines

\section{INTRODUCTION}

Recently, there has been tremendous growth in research on fuzzy automata theory, theoretically[8-10,12] as well as practically $[5,7,10,13]$. Fuzzy Mealy machine is a kind of fuzzy automaton with outputs capabilities based on both the current state and the current input on similar lines to fuzzy automaton, fuzzy Mealy machine generalize classical Mealy machines, in the sense of partial (degree of) transition of states and outputs.This makes possible to tackled uncertainty in transition as well output. Surprisingly, very little research has done in this area $[2,4,11]$. Algebraic study of fuzzy Mealy machines has done mainly by Mordeson et al. [11] and Jun Liu, Zhiwen Mo, Dong Qiu and Yang Wang [4]. Both the treatments study internal working of fuzzy Mealy machines such as equivalence and minimization. This kind of study for finite state machines has been discussed by Mordeson et. al. [8] and Kumbhojkar and Chaudhari[6]. In $[1,3]$ equivalence of fuzzy Mealy machine and fuzzy Moore machine is discussed. The aim of the present paper is to study fuzzy Mealy machine with the help of the topology induced by the successor operator defined on its state set. Here, submachines, (strongly) connected, cyclic (singly generated) and retrievable fuzzy Mealy machines with the help of this topology are studied. This study is analogous to that of topological study of fuzzy finite state machines discussed in [9]. Also products of fuzzy Mealy machines and their cyclicness, retrievablity, strongly connectedness, exchange property and connectedness are discussed. Precisely following results are obtained in this paper

(i) Fuzzy Mealy machine M is strongly connected if and only if the topology generated by the successor function is a discrete topology. (ii) Fuzzy Mealy machine M satisfies the exchange property $\Leftrightarrow$ it is union of its strongly connected submachines
$\Leftrightarrow$ it is retrievable (iii) Fuzzy Mealy machine M is strongly connected $\Leftrightarrow$ it is connected and retrievable $\Leftrightarrow$ its every submachine is strongly connected. It is also shown that the cartesian product and full direct product of fuzzy Mealy machines preserved these properties, where as the restricted direct product, cascade product and wreath product preserve these properties under strongly connectedness of individual fuzzy Mealy machine(s).

This paper consists of three sections. In section 2, successor function for the set of states of fuzzy Mealy machine is introduced. The concept of cyclic, retrievable, strongly connected, connected fuzzy Mealy machines along with their relationships are introduced. Sections 3, introduces various products of fuzzy Mealy machines such as cartesian product, full direct product, restricted direct product, cascade product, wreath product. The cyclicness, retrievability, and connectedness of these product are also discussed. The paper is concluded by giving future direction of research and by posing an open problem relating to the notion of topologies which are (always exists due to theorem (2.2)) for all the product introduced in this paper.

\section{FUZZY MEALY MACHINES}

Recall that a fuzzy Mealy machine (fmm) was introduced by Mordeson and Nair in [11] as a four tuple $M=$ $(Q, X, Y, \mu)$,where $\mathrm{Q}$ is a finite non-empty set of states, $\mathrm{X}$ is a finite non-empty set of inputs symbols, $\mathrm{Y}$ is a finite non-empty set of output symbols and $\mu$ is a fuzzy subset of $Q \times X \times Q \times Y$ i.e. $\mu: Q \times X \times Q \times Y \longrightarrow[0,1]$.

The extension of $\mu$ to $Q \times X^{*} \times Q \times Y^{*}$ is defined by

$$
\mu^{*}(q, \lambda, p, \lambda)= \begin{cases}1, & \text { if } q=p \\ 0, & \text { if } q \neq p\end{cases}
$$

$\mu^{*}(q, \lambda, p, b)=0$ and $\mu^{*}(q, x a, p, y b)=\bigvee\left\{\mu^{*}(q, x, r, y) \wedge\right.$ $\mu(r, a, p, b) \mid r \in Q\}, \forall q, p \in Q, \forall x \in X^{*}, \forall a \in X, \forall b \in$ $Y, \forall y \in Y^{*}$, where $\lambda$ denotes the empty string.

Then $\forall q, p \in Q, \forall x, u \in X^{*}, \forall y, v \in Y^{*}$ such that $|x|=|y|$ and $|u|=|v|$, we have $\mu^{*}(q, x u, p, y v)=\bigvee\left\{\mu^{*}(q, x, r, y) \wedge\right.$ $\left.\mu^{*}(r, u, p, v) \mid r \in Q\right\}$.

Therefore, $\forall q, p \in Q, \forall x \in X^{*}, \forall y \in Y^{*}$, if $|x| \neq|y|$ then $\mu^{*}(q, x, p, y)=0$. Due to this property, for the rest of the paper we shall assume that $|x|=|y|$ in any expression of the form $\mu^{*}(q, x, p, y)$ that may encounter.

We now introduce a topology on the state set of a given fuzzy Mealy machine. We begin with the concept of successor.

Definition 2.1 Let $M=(Q, X, Y, \mu)$ be a fmm. Let $q, p \in Q$. Then $p$ is called an immediate successor of $q$ if $\exists a \in X$ and $b \in Y$ such that $\mu(q, a, p, b)>0$ and $\mathrm{p}$ is called successor of $q$ if $\exists x \in X^{*}$ and $y \in Y^{*}$ such that $\mu^{*}(q, x, p, y)>0$. 
Notation 2.2 Let $M=(Q, X, Y, \mu)$ be a fmm and $q \in Q$. We shall denote $S(q)$ the set of all successor of $q$.

Definition 2.3 Let $M=(Q, X, Y, \mu)$ be a fmm and $T \subseteq Q$. The set of all successor of $T$, denoted by $S_{Q}(T)$, is defined to be the set $S_{Q}(T)=\bigcup\{S(q) \mid q \in T\}$.

When no confusion arises, we shall write $S(T)$ for $S_{Q}(T)$

Theorem 2.1 Let $M=(Q, X, Y, \mu)$ be a fmm. Define a relation $\sim$ on $\mathrm{Q}$ as $p \sim q$ if and only if $q$ is successor of $p$. Then $\sim$ is reflexive and transitive.

Theorem 2.2 Let $M=(Q, X, Y, \mu)$ be a fmm. Then the successor function, $S: \wp(Q) \longrightarrow \wp(Q)$, i.e. $S(A)$ the set of all successors of $A \subseteq Q$, is a Kuratowski's closure operator.

Proof. We prove idempotent property only. Clearly, $S(A) \subseteq$ $S(S(A))$. Let $q \in S(S(A))$. Then $q \in S(p)$ for some $p \in$ $S(A)$. Thus, $p \in S(r)$ for some $r \in A$. Now, $q$ is successor of $p$ and $p$ is successor $r$. Thus, $q \in S(r) \subseteq S(A)$, that is, $S(S(A)) \subseteq S(A)$. Hence $S(S(A))=S(A)$.

15mmTherefore, $\tau=\left\{A^{c} \mid S(A) \subseteq A\right\}$ defines a topology on Q. Thus, A subset A of Q is $\tau$-closed, if $S(A)=A$.

Clearly the set of all closed subset of $\mathrm{Q}$ is a poset under set inclusion operation and it is also a complete lattice with $\wedge S\left(A_{i}\right)=$ $\cap S\left(A_{i}\right)$ and $\vee S\left(A_{i}\right)=S\left(\cup\left(A_{i}\right)\right)$.

Definition 2.4 Let $M=(Q, X, Y, \mu)$ be a fmm. Then $N=$ $(T, X, Y, \nu)$ is called a submachine of $M$, if (1) $T$ is $\tau$-closed subset of Q and (2) $\mu_{\left.\right|_{T \times X \times T \times Y}}=\nu$. It is said to be proper submachine, if $\mathrm{T}$ is proper $\tau$-closed subset of $\mathrm{Q}$ and $\mu_{\mid T \times X \times T \times Y}=$ $\nu$.

Clearly, if $\mathrm{K}$ is a submachine of $N$ and $N$ is a submachine of $M$, then $\mathrm{K}$ is a submachine of $M$.

Definition 2.5 Let $M=(Q, X, Y, \mu)$ be a fmm. Let $q, p \in Q$ and $T \subseteq Q$. Suppose that if $p \in S(T \cup\{q\}), p \notin S(T)$, then $q \in S(T \cup\{p\})$. Then we say that $M$ satisfies the exchange property.

Theorem 2.3 Let $M=(Q, X, Y, \mu)$ be a fmm. Then $M$ satisfies the exchange property if and only if $\forall p, q \in Q, q \in S(p)$ if and only if $p \in S(q)$.

Proof. Let $p, q \in Q$ and $p \in S(q)$. Now, $p \notin S(\phi)$, therefore $q \in S(p)$. On similar line $q \in S(p)$ implies that $p \in S(q)$.

Conversely let $T \subseteq Q, p, q \in Q$. Suppose $p \in S(T \cup\{q\}), p \notin$ $S(T)$. Then $p \in S(q)$. Hence, $q \in S(p) \subseteq S(T \cup\{p\})$

Definition 2.6 Let $M=(Q, X, Y, \mu)$ be a fmm. Then $M$ is called strongly connected if $\forall p, q \in Q, p \in S(q)$

Theorem 2.4 Let $M=(Q, X, Y, \mu)$ be a fmm. Then $M$ is strongly connected if and only if $\tau$ is the discrete topology on Q.

Proof. Suppose $M$ is strongly connected. Let $N=(T, X, Y, \nu)$ be a submachine of $M$ such that $T \neq \phi$. Then $\exists q \in T$. Let $p \in Q$. Since $M$ is strongly connected, $p \in S(q)$. Hence, $T=Q$ and so $\tau$ is discrete topology on $\mathrm{Q}$.

Conversely if $p, q \in Q$ and $N=(S(q), X, Y, \nu)$, where $\nu=\mu_{\mid S(q) \times X \times S(q) \times Y}$, then $N$ is a submachine of $M$. Since $S(q) \neq \phi$ and $\tau$ is discrete, we have $S(q)=Q$. Thus, $p \in S(q)$. Hence, $M$ is strongly connected.

Theorem 2.5 Let $M=(Q, X, Y, \mu)$ be a fmm. Then for each $R \subseteq Q, S(R)$ is a $\tau$-closed set.

Proof. $S(S(R))=S(R)$ proves the theorem.

Thus, $N=\left(S(R), X, Y, \mu_{R}\right)$ is a submachine of $M$ for each $R \subseteq Q$, where $\mu_{R}=\mu_{\left.\right|_{S(R) \times X \times S(R) \times Y}}$.

Definition 2.7 Let $M=(Q, X, Y, \mu)$ be a fmm. Let $R \subseteq Q$. The smallest submachine generated by R, i.e. $\langle R\rangle$, is the intersection of all submachines of $\mathrm{M}$ whose state sets are subsets of Q containing R. Thus, $<R>=\left(\left(\bigcap_{i \in I} Q_{i}, X, Y, \bigcap_{i \in I} \mu_{i}\right)\right.$, where $N_{i}=\left(Q_{i}, X, Y, \mu_{i}\right)$ is a submachine of M such that $R \subseteq Q_{i}, \forall i$.

Note that the state set of $\langle R\rangle$ is the $\tau$-closure of $\mathrm{R}$.

Theorem 2.6 Let $M=(Q, X, Y, \mu)$ be a fmm. Let $R \subseteq Q$. Then $\langle R\rangle=\left(S(R), X, Y, \mu_{R}\right)$.
Proof. Now, $<R>=\left(\bigcap_{i \in I} Q_{i}, X, Y, \bigcap_{i \in I} \mu_{i}\right)$, where $N_{i}=$ $\left(Q_{i}, X, Y, \mu_{i}\right)$ are submachines of $M$ such that $R \subseteq Q_{i}$. It suffices to show that $S(R)=\bigcap Q_{i}$. Since $\left(S(R), X, Y, \mu_{R}\right)$ is a submachine of $M$ such that $R \subseteq S(R)$, we have that $S(R) \supseteq$ $\bigcap Q_{i}$. Let $p \in S(R)$. Then $\exists r \in R$ and $x \in X^{*}, y \in Y^{*}$ such $\bigcap_{i \in I}$

that $\mu^{*}(r, x, p, y)>0$. Now $r \in \bigcap_{i \in I} Q_{i}$ and since $\langle R>$ is a submachine of $M, p \in \bigcap_{i \in I} Q_{i}$. Thus $S(R) \subseteq \bigcap_{i \in I} Q_{i}$. Hence $S(R)=\bigcap_{i \in I} Q_{i}$

Definition 2.8 Let $M=(Q, X, Y, \mu)$ be a fmm. $M$ is called singly generated or cyclic if $\exists q \in Q$ such that $\{q\}$ is $\tau$-dense in Q. i.e $S(\{q\})=Q$. In this case $q$ is called a generator of $M$ and we say that $M$ is generated by $q$.

Hence, $M$ is singly generated by $q \in Q$ if and only if $M=$ $\langle\{q\}\rangle$.

Definition 2.9 A fmm $M=(Q, X, Y, \mu)$ is said to be retrievable when $\forall q \in Q, \forall x \in X^{*}, y \in Y^{*}$, if $\exists p \in Q$ such that $\mu^{*}(q, x, p, y)>0$, then $\exists u \in X^{*}, v \in Y^{*}$ such that $\mu^{*}(p, u, q, v)>0$.

Theorem 2.7 Let $M=(Q, X, Y, \mu)$ be a fmm. Then the following statements are equivalent

1. $M$ satisfies the exchange property

2. $M$ is union of strongly connected submachines

3. $M$ is retrievable

Proof. (1) $\Rightarrow$ (2): Clearly $M=\bigcup_{i=1}^{n}<\left\{q_{i}\right\}>$, where $S\left(\left\{q_{1}, q_{2}, \ldots, q_{n}\right\}\right)=Q$. Also $S\left(q_{i}\right) \cap S\left(q_{j}\right)=\phi$ if $i \neq j$. Let $p, q \in S\left(q_{i}\right)$. Then $q_{i} \in S(p)$ and so $q \in S(p)$. Thus $\left\langle q_{i}\right\rangle$ is strongly connected. (2) $\Rightarrow(1)$ : Now $M=\bigcup_{i=1}^{n} M_{i}$, where each $M_{i}=\left(Q_{i}, X, Y, \mu_{i}\right)$ strongly connected. Let $p, q \in Q$. Suppose $p \in S(q)$. Now $\exists i$ such that $q \in Q_{i}$. Then $p \in S(q) \subseteq S\left(Q_{i}\right)=$ $Q_{i}$. Thus $p, q \in Q_{i}$. Since $M_{i}$ is strongly connected, $q \in S(p)$. Hence $M$ satisfies the Exchange Property by theorem(2.3). (2) $\Rightarrow$ (3): Now $M=\bigcup_{i=1}^{n} M_{i}$, where each $M_{i}=$ $\left(Q_{i}, X, Y, \mu_{i}\right)$ strongly connected. Let $q \in Q, u \in X^{*}, v \in$ $Y^{*}$ be such that $\mu^{*}(q, u, t, v)>0$ for some $t \in Q$. Now $q \in Q_{i}$ for some $i$. Thus $t \in S(q) \subseteq S\left(Q_{i}\right)$. Since $M_{i}$ is strongly connected, $q \in S(t)$. Hence $\exists x \in X^{*}, y \in$ $Y^{*}$ such that $\mu^{*}(t, x, q, y)>0$. Thus $M$ is retrievable. (3) $\Rightarrow$ (2): Let $q \in Q$ and let $r, t \in S(q)$. Then $\exists x, u \in X^{*}$ and $\exists y, v \in Y^{*}$ such that $\mu^{*}(q, x, r, u)>0$ and $\mu^{*}(q, u, r, v)>0$. Since $M$ is retrievable $\exists z \in X^{*}$ and $w \in Y^{*}$ such that $\mu^{*}(r, z, q, w)>0$. Hence $q \in S(r)$ and $\langle q>$ is strongly connected. So $M=\bigcup_{q \in Q}<q>$.

Definition 2.10 Let $M=(Q, X, Y, \mu)$ be a fmm. A proper submachine $N=(T, X, Y, \nu)$ is said to be separated if Q-T is a proper $\tau$-closed subset of $\mathrm{Q}$.

Theorem 2.8 Let $M=(Q, X, Y, \mu)$ be a fmm. Let $N=$ $(T, X, Y, \nu)$ be a submachine of $M$. Then $N$ is separated if and only if $S(Q-T) \cap T=\phi$.

Proof. Suppose $N$ is separated. Then T is $\tau$-closed subset of Q. Thus, $\mathrm{S}(\mathrm{Q}-\mathrm{T})=\mathrm{Q}-\mathrm{T}$. Hence $S(Q-T) \cap T=Q-T \cap T=\phi$. Conversely, let $q \in S(Q-T)$. Then by assumption $q \notin T$. Thus, $q \in Q-T$. Therefore, $S(Q-T)=Q-T$. i.e. $Q-T$ is a $\tau$ closed subset of Q.

Theorem 2.9 Let $M=(Q, X, Y, \mu)$ be a cyclic then it has separated and strongly connected submachine.

Proof. Take $T=\{p \in Q \mid S(p)=Q\}$. Then $N=$ $\left(T, X, Y,\left.\mu\right|_{S(q)}\right)$ is the required submachine.

Definition 2.11 Let $M=(Q, X, Y, \mu)$ be a fmm. Then $M$ is said to be connected if and only if $Q$ has no proper $\tau$-open and $\tau$-closed subset.

Theorem 2.10 Let $M=(Q, X, Y, \mu)$ be a fmm. Then $M$ is connected if and only if $M$ has no separated submachine.

Proof. Suppose $M$ is connected. Let if possible $M$ has a proper 
separated submachine, say $N=(T, X, Y, \nu)$. Then $T$ is a proper $\tau$-closed subset of $Q$. Since, $N$ is separated, we have $S(Q-T)=Q-T$. Therefore, $Q_{T}$ is $\tau$-closed. i.e. $T$ is $\tau$ open subset of $Q$. Therefore, $T$ is proper $\tau$-closed and $\tau$-open subset of $Q$ which is contradiction to $M$ is connected.

Conversely, if $M$ is not connected then $M$ has a proper separated submachine, say $N=(T, X, Y, \nu)$. Then clearly, $T$ is a proper $\tau$-open and $\tau$-closed subset of $\mathrm{Q}$, which is contradiction to the hypothesis. Therefore, $M$ is must not connected.

The difference between strongly connected and connected is depicted in the following example.

Example 2.11 Consider a Mealy machine $M: \mu\left(p, x_{2}, q, y_{2}\right)=$ $0.3, \mu\left(p, x_{1}, r, y_{1}\right)=0.7$ and $\mu\left(q, x_{1}, r, y_{1}\right)=0.8$.

Then $M$ is connected but not strongly connected.

Theorem 2.12 Let $M=(Q, X, Y, \mu)$ be a fmm. Then $M$ is connected if and only if for all proper submachines $N=$ $(T, X, Y, \nu), \exists s \in Q-T$ and $t \in T$ such that $S(s) \cap S(t) \neq \phi$. Theorem 2.13 Let $M$ be strongly connected fmm. Then $M$ is cyclic, retrievable, connected.

Theorem 2.14 Let $M=(Q, X, Y, \mu)$ be a fmm. Then following assertions are equivalent.

1. $M$ is strongly connected.

2. $M$ is connected and retrievable.

3. Every submachine of $M$ is strongly connected.

Proof. (1) $\Rightarrow(2)$ : By theorem (2.4), $M$ does not have any proper submachine and so $M$ has no proper separated submachines. Thus $M$ is connected. Now we show that $M$ is retrievable. Let $q, t \in Q$ and $x \in X^{*}, y \in Y^{*}$ be such that $\mu^{*}(q, x, t, y)>0$. Since $M$ is strongly connected $q \in S(t)$. Then $\exists u \in X^{*}, v \in Y^{*}$ such that $\mu^{*}(t, u, q, v)>0$. Hence $M$ is retrievable.

(2) $\Rightarrow$ (3): Let $N=(T, X, Y, \nu)$ be a submachine of $M$. Suppose $p, q \in T$ are such that $p \notin S(q)$. Then $S(q) \neq Q$ and so $K=(S(q), X, Y, \mu \mid S(q) \times X \times S(q) \times Y)$ is a proper submachine of $M$. Since $M$ is connected, $S(Q-S(q)) \cap S(q) \neq \phi$. Let $r \in S(Q-S(q)) \cap S(q)$. Then $r \in S(t)$ for some $t \in Q-S(q)$ and $r \in S(q)$. Now $\exists x \in X^{*}$ andy $\in Y^{*}$ such that $\left.\mu^{*}(t, x, r, y)>0\right)$. Since $M$ is retrievable, $\exists u \in X^{*}$ and $v \in Y^{*}$ such that $\left.\mu^{*}(r, u, t, v)>0\right)$. Thus $t \in S(r)$. Hence $t \in S(r) \subseteq S(q)$, a contradiction. Thus $p \in S(q) \forall p, q \in T$. Hence $N$ is strongly connected.

(3) $\Rightarrow(1)$ : Obvious.

Theorem 2.15 If $M$ is cyclic and $T=\{p \in Q \mid S(p)=Q\}$ then $N=\left(T, X, Y, \mu_{\left.\right|_{S(q)}}\right)$ has a retrievable and connected submachine.

Proof. Follows by above theorem (2.13).

\section{PRODUCTS OF FUZZY MEALY MACHINES}

This section is an introduction of various products of fmms and discussed their interrelationship in terms of cyclicness, retrievability exchange property and connectedness.

Definition 3.1 Let $M_{i}=\left(Q_{i}, X_{i}, Y_{i}, \mu_{i}\right)$ be a fmms, $i=1,2$. Then the machine $M_{1} \odot M_{2}=\left(Q, X, Y, \mu_{1} \odot \mu_{2}\right)$ is called 1) the cartesian product of fmms of $M_{1}$ and $M_{2}$, with $X_{1} \cap$ $X_{2}=\phi$ and $Y_{1} \cap Y_{2}=\phi$, if $Q=Q_{1} \times Q_{2}, X=X_{1} \cup X_{2}, Y=$ $Y_{1} \cup Y_{2}$ and $\left(\mu_{1} \odot \mu_{2}\right)\left(\left(q_{1}, q_{2}\right), a,\left(p_{1}, p_{2}\right), b\right)=$

$$
= \begin{cases}\mu_{1}\left(q_{1}, a, p_{1}, b\right) & \text { if } a \in X_{1}, b \in Y_{1} \text { and } q_{2}=p_{2} \\ \mu_{2}\left(q_{2}, a, p_{2}, b\right) & \text { if } a \in X_{2}, b \in Y_{2} \text { and } q_{1}=p_{1} \\ 0 & \text { otherwise }\end{cases}
$$

$\forall\left(p_{1}, p_{2}\right),\left(q_{1}, q_{2}\right) \in Q_{1} \times Q_{2}, a \in X_{1} \cup X_{2}, b \in Y_{1} \cup Y_{2}$. 2) the full direct product of fmms of $M_{1}$ and $M_{2}$, if $Q=Q_{1} \times Q_{2}, X=X_{1} \times X_{2}, Y=Y_{1} \times Y_{2}$ and $\left(\mu_{1} \odot \mu_{2}\right)^{*}\left(\left(q_{1}, q_{2}\right),\left(x_{1}, x_{2}\right),\left(p_{1}, p_{2}\right),\left(y_{1}, y_{2}\right)\right)=$ $\mu_{1}^{*}\left(q_{1}, x_{1}, p_{1}, y_{1}\right) \wedge \mu_{2}^{*}\left(q_{2}, x_{2}, p_{2}, y_{2}\right), \forall\left(q_{1}, q_{2}\right),\left(p_{1}, p_{2}\right) \in$
$Q_{1} \times Q_{2}, \forall\left(x_{1}, x_{2}\right) \in X_{1} \times X_{2}, \forall\left(y_{1}, y_{2}\right) \in Y_{1} \times Y_{2}$.

3) the restricted direct product of fmms of $M_{1}$ and $M_{2}$, if $Q=Q_{1} \times Q_{2}, X=X_{1}=X_{2}, Y=Y_{1}=Y_{2}$ and $\left(\mu_{1} \odot \mu_{2}\right)^{*}\left(\left(q_{1}, q_{2}\right), x,\left(p_{1}, p_{2}\right), y\right)=\mu_{1}^{*}\left(q_{1}, x, p_{1}, y\right) \wedge$ $\mu_{2}^{*}\left(q_{2}, x, p_{2}, y\right), \forall\left(q_{1}, q_{2}\right),\left(p_{1}, p_{2}\right) \in Q_{1} \times Q_{2}, \forall x \in X, \forall y \in$ $Y$

4)the cascade product of fmms of $M_{1}$ and $M_{2}$, if $Q=Q_{1} \times Q_{2}, X=X_{2}, Y=Y_{2}$

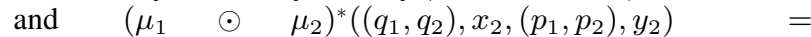
$\mu_{1}^{*}\left(q_{1}, \omega_{x}\left(q_{2}, x_{2}\right), p_{1}, \omega_{y}\left(q_{2}, y_{2}\right)\right) \quad \wedge \mu_{2}^{*}\left(q_{2}, x_{2}, p_{2}, y_{2}\right)$, $\forall\left(q_{1}, q_{2}\right),\left(p_{1}, p_{2}\right) \in Q_{1} \times Q_{2}, \forall x_{2} \in X_{2}$ and $\forall y_{2} \in Y_{2}$ and $\omega_{x}: Q_{2} \times X_{2} \rightarrow X_{1}$ and $\omega_{y}: Q_{2} \times Y_{2} \rightarrow Y_{1}$.

5) the wreath product of fmms of $M_{1}$ and $M_{2}$, if $Q=Q_{1} \times Q_{2}, X=X_{1}^{Q_{2}} \times X_{2}, Y=Y_{1}^{Q_{2}} \times Y_{2}$ and $\left(\mu_{1} \odot \mu_{2}\right)^{*}\left(\left(q_{1}, q_{2}\right),\left(f, x_{2}\right),\left(p_{1}, p_{2}\right),\left(g, y_{2}\right)\right)=\mu_{1}^{*}\left(q_{1}, f\left(q_{2}\right)\right.$, $\left.p_{1}, g\left(q_{2}\right)\right) \quad \wedge \mu_{2}^{*}\left(q_{2}, x_{2}, p_{2}, y_{2}\right), \forall\left(q_{1}, q_{2}\right),\left(p_{1}, p_{2}\right) \quad \in$ $Q_{1} \times Q_{2}, \forall x_{2} \in X_{2}$ and $\forall y_{2} \in Y_{2}$ and $X_{1}^{Q_{2}}=\{f$ : $\left.Q_{2} \rightarrow X_{1}\right\}$ and $Y_{1}^{Q_{2}}=\left\{g: Q_{2} \rightarrow Y_{1}\right\}$.

Remark 3.1 i) The restricted direct product of $M_{1}$ and $M_{2}$ is a special case of their cascade product, $X_{1}=X_{2}, Y_{1}=Y_{2}$ and $\omega_{x}: Q_{2} \times X_{2} \rightarrow X_{1}, \omega_{y}: Q_{2} \times Y_{2} \rightarrow Y_{1}$ both are projection functions.

ii) The cascade product of $M_{1}$ and $M_{2}$ is a special case of their wreath product, when $X_{1}^{Q_{2}}=\left\{\omega_{x}\right\}$ and $Y_{1}^{Q_{2}}=\left\{\omega_{y}\right\}$.

Theorem 3.2 Let $M_{i}=\left(Q_{i}, X_{i}, Y_{i}, \mu_{i}\right)$ be a fmms, $i=1,2$ and let $X_{1} \cap X_{2}=\phi$ and $Y_{1} \cap Y_{2}=\phi$. Let $M_{1} \odot M_{2}=\left(Q_{1} \times Q_{2}, X_{1} \cup X_{2}, Y_{1} \cup Y_{2}, \mu_{1} \odot \mu_{2}\right)$

be the cartesian product of $M_{1}$ and $M_{2}$. Then $\forall x \in$ $X_{1}^{*} \cup X_{2}^{*}, x \neq \lambda, \forall y \in Y_{1}^{*} \cup Y_{2}^{*}, y \neq \lambda$

$\left(\mu_{1} \odot \mu_{2}\right)^{*}\left(\left(q_{1}, q_{2}\right), x,\left(p_{1}, p_{2}\right), y\right)=$

$$
= \begin{cases}\mu_{1}^{*}\left(q_{1}, x, p_{1}, y\right) & \text { if } x \in X_{1}^{*}, y \in Y_{1}^{*} \text { and } q_{2}=p_{2} \\ \mu_{2}^{*}\left(q_{2}, x, p_{2}, y\right) & \text { if } x \in X_{2}^{*}, y \in Y_{2}^{*} \text { and } q_{1}=p_{1} \\ 0 & \text { otherwise }\end{cases}
$$

$\forall\left(p_{1}, p_{2}\right),\left(q_{1}, q_{2}\right) \in Q_{1} \times Q_{2}$

Proof. Let $x \in X_{1}^{*} \cup X_{2}^{*}, x \neq \lambda, y \in Y_{1}^{*} \cup Y_{2}^{*}, y \neq \lambda$ and let $|x|=|y|=n$. Suppose that $x \in X_{1}^{*}$ and $y \in Y_{1}^{*}$. Clearly the result is true if $n=1$. Suppose the result is true $\forall u \in X_{1}^{*}$, $u \mid=n-1, n>1$ and $\forall v \in Y_{1}^{*},|v|=n-1, n>1$. Let $x=a u$ where $a \in X_{1}$ and $u \in X_{1}^{*}$ and $y=b v$ where $b \in Y_{1}$ and $v \in Y_{1}^{*}$. Now, $\left(\mu_{1} \odot \mu_{2}\right)^{*}\left(\left(p_{1}, p_{2}\right), a u,\left(q_{1}, q_{2}\right), b v\right)=$

$$
\begin{aligned}
= & \vee\left\{\left(\mu_{1} \odot \mu_{2}\right)\left(\left(p_{1}, p_{2}\right), a,\left(r_{1}, r_{2}\right), b\right)\right. \\
& \wedge\left(\mu_{1} \odot \mu_{2}\right)^{*}\left(\left(r_{1}, r_{2}\right), u,\left(q_{1}, q_{2}\right), v\right) \mid \\
= & \vee\left\{\mu_{1}, r_{2}\left(p_{1}, a, Q_{1} \times Q_{1}, b\right) \wedge\left(\mu_{1} \odot \mu_{2}\right)^{*}\left(\left(r_{1}, p_{2}\right), u,\left(q_{1}, q_{2}\right), v\right)\right. \\
& \left.\mid r_{1} \in Q_{1}\right\} \\
= & \begin{cases}\vee\left\{\mu_{1}\left(p_{1}, a, r_{1}, b\right) \wedge \mu_{1}^{*}\left(r_{1}, u, q_{1}, v\right) \mid r_{1} \in Q_{1}\right\} & \text { if } p_{2}=q_{2} \\
0 & \text { otherwise }\end{cases} \\
= & \begin{cases}\mu_{1}^{*}\left(p_{1}, a u, q_{1}, b v\right) & \text { if } p_{2}=q_{2} \\
0 & \text { otherwise }\end{cases}
\end{aligned}
$$

The result is now follows by induction. Similarly, if $x \in X_{2}^{*}$ and $y \in Y_{2}^{*}$ one can prove the other case.

Theorem 3.3 Let $M_{i}=\left(Q_{i}, X_{i}, Y_{i}, \mu_{i}\right)$ be a fmms, $i=1,2$ and let $X_{1} \cap X_{2}=\phi$ and $Y_{1} \cap Y_{2}=\phi$. Let $M_{1} \odot M_{2}=\left(Q_{1} \times Q_{2}, X_{1} \cup X_{2}, Y_{1} \cup Y_{2}, \mu_{1} \odot \mu_{2}\right)$ be the cartesian product of $M_{1}$ and $M_{2}$. Then $\forall x_{1} \in X_{1}, x_{2} \in X_{2}, y_{1} \in Y_{1}, y_{2} \in Y_{2}$ $\left(\mu_{1} \odot \mu_{2}\right)^{*}\left(\left(q_{1}, q_{2}\right), x_{1} x_{2},\left(p_{1}, p_{2}\right), y_{1} y_{2}\right)=$ $\mu_{1}^{*}\left(q_{1}, x_{1}, p_{1}, y_{1}\right) \wedge \mu_{2}^{*}\left(q_{2}, x_{2}, p_{2}, y_{2}\right)$ $=\left(\mu_{1} \odot \mu_{2}\right)^{*}\left(\left(q_{1}, q_{2}\right), x_{2} x_{1},\left(p_{1}, p_{2}\right), y_{2} y_{1}\right)$ 
Proof. Let $x_{1} \in X_{1}^{*}, x_{2} \in X_{2}^{*}, y_{1} \in Y_{1}^{*}, y_{2} \in Y_{2}^{*}$ and $\left(q_{1}, q_{2}\right),\left(p_{1}, p_{2}\right) \in Q_{1} \times Q_{2}$. case(i) If $x_{1}=x_{2}=y_{1}=y_{2}=\lambda$ then $x_{1} x_{2}=y_{1} y_{2}=\lambda$. case(ii) Suppose, $\left(q_{1}, q_{2}\right)=\left(p_{1}, p_{2}\right)$, then $q_{1}=p_{1}$ or $q_{2}=p_{2}$. Hence, $\left(\mu_{1} \odot \mu_{2}\right)\left(\left(q_{1}, q_{2}\right), x_{1} x_{2},\left(p_{1}, p_{2}\right), y_{1} y_{2}\right)=1=1 \wedge 1=$ $\mu_{1}^{*}\left(q_{1}, x_{1}, p_{1}, y_{1}\right) \wedge \mu_{2}^{*}\left(q_{2}, x_{2}, p_{2}, y_{2}\right)$.

suppose, $\left(q_{1}, q_{2}\right) \neq\left(p_{1}, p_{2}\right)$, then either $q_{1} \neq p_{1}$ and $q_{2} \neq p_{2}$. Thus, $\mu_{1}^{*}\left(q_{1}, x_{1}, p_{1}, y_{1}\right) \wedge \mu_{2}^{*}\left(q_{2}, x_{2}, p_{2}, y_{2}\right)=0$. Hence $\left(\mu_{1} \odot \mu_{2}\right)\left(\left(q_{1}, q_{2}\right), x_{1} x_{2},\left(p_{1}, p_{2}\right), y_{1} y_{2}\right)=0=$ $\mu_{1}^{*}\left(q_{1}, x_{1}, p_{1}, y_{1}\right) \wedge \mu_{2}^{*}\left(q_{2}, x_{2}, p_{2}, y_{2}\right)$.

case(iii) If $x_{1}=\lambda, y_{1}=\lambda$ and $x_{2} \neq \lambda, y_{2} \neq \lambda$ or $x_{1} \neq \lambda, y_{1} \neq \lambda$ and $x_{2}=\lambda, y_{2}=\lambda$.Then by theorem 3.2 result holds.

case(iv) Suppose, $x_{1} \neq \lambda, y_{1} \neq \lambda$ and $x_{2} \neq \lambda, y_{2} \neq \lambda$. Now $\left(\mu_{1} \odot \mu_{2}\right)^{*}\left(\left(q_{1}, q_{2}\right), x_{1} x_{2},\left(p_{1}, p_{2}\right), y_{1} y_{2}\right)=$

$$
\begin{aligned}
= & \bigvee\left\{\left(\mu_{1} \odot \mu_{2}\right)^{*}\left(\left(q_{1}, q_{2}\right), x_{1},\left(r_{1}, r_{2}\right), y_{1}\right)\right. \\
& \wedge\left(\mu_{1} \odot \mu_{2}\right)^{*}\left(\left(r_{1}, r_{2}\right), x_{2},\left(p_{1}, p_{2}\right), y_{2}\right) \mid \\
& \left.\left(r_{1}, r_{2}\right) \in Q_{1} \times Q_{2}\right\} \\
= & \bigvee\left\{\bigvee \left\{\left(\mu_{1} \odot \mu_{2}\right)^{*}\left(\left(q_{1}, q_{2}\right), x_{1},\left(r_{1}, r_{2}\right), y_{1}\right)\right.\right. \\
& \left.\wedge\left(\mu_{1} \odot \mu_{2}\right)^{*}\left(\left(r_{1}, r_{2}\right), x_{2},\left(p_{1}, p_{2}\right), y_{2}\right) \mid r_{2} \in Q_{2}\right\} \mid \\
& \left.r_{1} \in Q_{1}\right\} \\
= & \bigvee\left\{\left(\mu_{1} \odot \mu_{2}\right)^{*}\left(\left(q_{1}, q_{2}\right), x_{1},\left(r_{1}, q_{2}\right), y_{1}\right)\right. \\
& \left.\wedge\left(\mu_{1} \odot \mu_{2}\right)^{*}\left(\left(r_{1}, q_{2}\right), x_{2},\left(p_{1}, p_{2}\right), y_{2}\right) \mid r_{1} \in Q_{1}\right\} \\
= & \mu_{1}^{*}\left(q_{1}, x_{1}, p_{1}, y_{1}\right) \wedge \mu_{2}^{*}\left(q_{2}, x_{2}, p_{2}, y_{2}\right)
\end{aligned}
$$

Similarly, $\left(\mu_{1} \odot \mu_{2}\right)^{*}\left(\left(q_{1}, q_{2}\right), x_{2} x_{1},\left(p_{1}, p_{2}\right), y_{2} y_{1}\right) \quad=$ $\mu_{1}^{*}\left(q_{1}, x_{1}, p_{1}, y_{1}\right) \wedge \mu_{2}^{*}\left(q_{2}, x_{2}, p_{2}, y_{2}\right)$.

Theorem 3.4 Let $M_{i}=\left(Q_{i}, X_{i}, Y_{i}, \mu_{i}\right)$ be a fmms, $i=1,2$ and let $X_{1} \cap X_{2}=\phi$ and $Y_{1} \cap Y_{2}=\phi$. Let $M_{1} \odot M_{2}=\left(Q_{1} \times Q_{2}, X_{1} \cup X_{2}, Y_{1} \cup Y_{2}, \mu_{1} \odot \mu_{2}\right)$

be the cartesian product of $M_{1}$ and $M_{2}$. Then $\forall x \in$ $\left(X_{1} \cup X_{2}\right)^{*} \exists x_{1} \in X_{1}^{*}, x_{2} \in X_{2}^{*}$ and $\forall y \in\left(Y_{1} \cup Y_{2}\right)^{*} \exists y_{1} \in$ $Y_{1}^{*}, y_{2} \in Y_{2}^{*}$ such that $\left(\mu_{1} \odot \mu_{2}\right)^{*}\left(\left(q_{1}, q_{2}\right), x,\left(p_{1}, p_{2}\right), y\right)=$ $\left(\mu_{1} \odot \mu_{2}\right)^{*}\left(\left(q_{1}, q_{2}\right), x_{1} x_{2},\left(p_{1}, p_{2}\right), y_{1} y_{2}\right)$

$\forall\left(p_{1}, p_{2}\right),\left(q_{1}, q_{2}\right) \in Q_{1} \times Q_{2}$.

Proof. Let $x \in\left(X_{1} \cup X_{2}\right)^{*}, y \in\left(Y_{1} \cup Y_{2}\right)^{*}$ and $\left(p_{1}, p_{2}\right),\left(q_{1}, q_{2}\right) \in Q_{1} \times Q_{2}$. If $x=\lambda=y$, then we can choose $x_{1}=x_{2}=y_{1}=y_{2}=\lambda$. In this case the result is trivially true. Suppose $x \neq \lambda, y \neq \lambda$. If $x \in X_{1}^{*}$ or $x \in X_{2}^{*}, y \in Y_{1}^{*}$ or $y \in Y_{2}^{*}$, then again the result is trivially true. Suppose $x \notin X_{1}^{*} x \notin X_{2}^{*}$ and $y \notin Y_{1}^{*}$ or $y \notin Y_{2}^{*}$

case(I) If $x=x_{1} x_{2}, x_{1} \in X_{1}^{*}-\lambda, x_{2} \in X_{2}^{*}-\lambda$ and $y=y_{1} y_{2}, y_{1} \in Y_{1}^{*}-\lambda, y_{2} \in Y_{2}^{*}-\lambda$. Then result follows by Theorem (3.3)

case(II) Suppose $x=x_{11} x_{21} x_{12}$ where $x_{11}, x_{12} \in$ $X_{1}^{*}, x_{21} \in X_{2}^{*}$ and $y=y_{11} y_{21} y_{12}$, where $y_{11}, y_{12} \in$ $Y_{1}^{*}, y_{21} \in Y_{2}^{*}, x_{1 i}, x_{21}, y_{1 i}, y_{21}$ are non-empty strings, $i=1,2$. Let $x_{1}=x_{11} x_{12} \in X_{1}^{*}$ and $x_{2}=x_{21}$ and $y_{1}=y_{11} y_{12} \in Y_{1}^{*}$ and $y_{2}=y_{21}$. Then $\left(\mu_{1} \odot \mu_{2}\right)^{*}\left(\left(q_{1}, q_{2}\right), x_{11} x_{21} x_{12},\left(p_{1}, p_{2}\right), y_{11} y_{21} y_{12}\right)=$ $\bigvee\left\{\left(\mu_{1} \odot \mu_{2}\right)^{*}\left(\left(q_{1}, q_{2}\right), x_{11},\left(r_{1}, r_{2}\right), y_{11}\right) \quad \wedge\left(\mu_{1} \odot\right.\right.$ $\left.\left.\mu_{2}\right)^{*}\left(\left(r_{1}, r_{2}\right), x_{21} x_{12},\left(p_{1}, p_{2}\right), y_{21} y_{12}\right) \mid\left(r_{1}, r_{2}\right) \in Q_{1} \times Q_{2}\right\}$ $=\bigvee\left\{\left(\mu_{1} \odot \mu_{2}\right)^{*}\left(\left(q_{1}, q_{2}\right), x_{11},\left(r_{1}, r_{2}\right), \quad y_{11}\right) \wedge\left(\mu_{1} \odot\right.\right.$ $\left.\left.\mu_{2}\right)^{*}\left(\left(r_{1}, r_{2}\right), x_{12} x_{21},\left(p_{1}, p_{2}\right), y_{12} y_{21}\right) \mid\left(r_{1}, r_{2}\right) \in Q_{1} \times Q_{2}\right\}$ $=\left(\mu_{1} \odot \mu_{2}\right)^{*}\left(\left(q_{1}, q_{2}\right), x_{11} x_{12} x_{21},\left(p_{1}, p_{2}\right), y_{11} y_{12} y_{21}\right)$ case(III) Suppose $x=x_{21} x_{11} x_{22}$ where $x_{21}, x_{22} \in X_{2}^{*}, x_{11} \in$ $X_{1}^{*}$ and $y=y_{21} y_{11} y_{22}$ where $y_{21}, y_{22} \in Y_{2}^{*}, y_{11} \in Y_{1}^{*}$, $x_{21}, x_{2 i}, y_{21}, y_{2 i}$ are non-empty strings $i=1,2$. Let $x_{2}=x_{21} x_{22} \in X_{2}^{*}$ and $x_{1}=x_{11}$ and $y_{2}=y_{21} y_{22} \in Y_{2}^{*}$ and $y_{1}=y_{11}$. The proof of this case is similar to case(II)

case(IV) Suppose $x=x_{11} x_{21} x_{12} x_{22}$, where $x_{11}, x_{12} \in$ $X_{1}^{*}, x_{21}, x_{22} \in X_{2}^{*}$ and $y=y_{11} y_{21} y_{12} y_{22}$, where $y_{11}, y_{12} \in Y_{1}^{*}, y_{21}, y_{22} \in Y_{2}^{*}, x_{1 i}, x_{2 i}, y_{1 i}, y_{2 i}$ are non-empty strings, $i=1,2$. Let $x_{1}=x_{11} x_{12} \in X_{1}^{*} x_{2}=x_{21} x_{22} \in X_{2}^{*}$ and $y_{1}=y_{11} y_{12} \in Y_{1}^{*} y_{2}=y_{21} y_{22} \in Y_{2}^{*}$

Then $\left(\mu_{1} \odot \mu_{2}\right)^{*}\left(\left(q_{1}, q_{2}\right), x_{11} x_{21} x_{12} x_{22},\left(p_{1}, p_{2}\right), y_{11} y_{21} y_{12} y_{22}\right)=$ $\bigvee\left\{\left(\mu_{1} \odot \mu_{2}\right)^{*}\left(\left(q_{1}, q_{2}\right), x_{11},\left(r_{1}, r_{2}\right), \quad y_{11}\right) \wedge\left(\mu_{1} \odot\right.\right.$ $\left.\mu_{2}\right)^{*}\left(\left(r_{1}, r_{2}\right), x_{21} x_{12} x_{22}, \quad\left(p_{1}, p_{2}\right), y_{21} y_{12} y_{22}\right) \mid\left(r_{1}, r_{2}\right) \quad \in$ $\left.Q_{1} \times Q_{2}\right\}=\bigvee\left\{\left(\mu_{1} \odot \mu_{2}\right)^{*}\left(\left(q_{1}, q_{2}\right), x_{11},\left(r_{1}, r_{2}\right), y_{11}\right) \wedge\left(\mu_{1} \odot\right.\right.$ $\left.\mu_{2}\right)^{*}\left(\left(r_{1}, r_{2}\right), x_{12} x_{21} x_{22},\left(p_{1}, p_{2}\right), y_{12} y_{21} y_{22}\right) \mid\left(r_{1}, r_{2}\right) \quad \in$

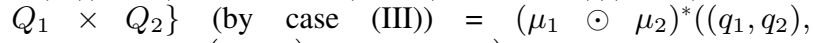
$\left.x_{11} x_{12} x_{21} x_{22},\left(p_{1}, p_{2}\right), y_{11} y_{12} y_{21} y_{22}\right)$.

case(V)Suppose $x=x_{21} x_{11} x_{22} x_{12}$, where $x_{11}, x_{12} \in$ $X_{1}^{*}, x_{21}, x_{22} \in X_{2}^{*}$ and $y=y_{21} y_{11} y_{22} y_{12}$, where $y_{11}, y_{12} \in Y_{1}^{*}, y_{21}, y_{22} \in Y_{2}^{*}, x_{1 i}, x_{2 i}, y_{1 i}, y_{2 i}$ are non-empty strings, $i=1,2$. Let $x_{1}=x_{11} x_{12} \in X_{1}^{*} x_{2}=x_{21} x_{22} \in X_{2}^{*}$ and $y_{1}=y_{11} y_{12} \in Y_{1}^{*} y_{2}=y_{21} y_{22} \in Y_{2}^{*}$. The proof of this case is similar to case(IV). The theorem now follows by induction

Theorem 3.5 Let $M_{i}=\left(Q_{i}, X_{i}, Y_{i}, \mu_{i}\right)$ be a fmms, $i=1,2$. Then fmms $M_{1} \odot M_{2}$ is cyclic if and only if $M_{1}$ and $M_{2}$ are cyclic, where $\odot$ is cartesian product and full direct product.

Proof. 1) When $\odot$ is cartesian product. Suppose $M_{1}$ and $M_{2}$ are cyclic, say $Q_{1}=S\left(q_{1}\right)$ and $Q_{2}=S\left(q_{2}\right)$ for some $q_{1} \in Q_{1}$ and $q_{2} \in Q_{2}$. Let $\left(p_{1}, p_{2}\right) \in Q_{1} \times Q_{2}$. Then $\exists x_{1} \in X_{1}^{*}, x_{2} \in X_{2}^{*}, y_{1} \in Y_{1}^{*}, y_{2} \in Y_{2}^{*}$ such that $\mu_{1}^{*}\left(q_{1}, x_{1}, p_{1}, y_{1}\right)>0$ and $\mu_{2}^{*}\left(q_{2}, x_{2}, p_{2}, y_{2}\right)>0$. Thus $\left(\mu_{1} \odot \mu_{2}\right)^{*}\left(\left(q_{1}, q_{2}\right), x_{1} x_{2},\left(p_{1}, p_{2}\right), y_{1} y_{2}\right)=$ $\mu_{1}^{*}\left(q_{1}, x_{1}, p_{1}, y_{1}\right) \wedge \mu_{2}^{*}\left(q_{2}, x_{2}, p_{2}, y_{2}\right)>0$. That is, $\left(p_{1}, p_{2}\right) \in S\left(q_{1}, q_{2}\right)$. Thus, $Q_{1} \times Q_{2}=S\left(\left(q_{1}, q_{2}\right)\right)$. Hence $M_{1} \odot M_{2}$ is cyclic.

Conversely, Suppose $M_{1} \odot M_{2}$ is cyclic. Let $Q_{1} \times Q_{2}=$ $S\left(\left(q_{1}, q_{2}\right)\right)$, for some $\left(q_{1}, q_{2}\right) \in Q_{1} \times Q_{2}$. Let $p_{1} \in Q_{1}$ and $p_{2} \in Q_{2}$. Then $\exists x \in\left(X_{1} \cup X_{2}\right)^{*}$ and $\exists y \in\left(Y_{1} \cup Y_{2}\right)^{*}$ such that $\left(\mu_{1} \odot \mu_{2}\right)^{*}\left(\left(q_{1}, q_{2}\right), x,\left(p_{1}, p_{2}\right), y\right)>0$. Then by theorem $3.3 \exists x_{1} \in X_{1}^{*}, x_{2} \in X_{2}^{*}$ and $\exists y_{1} \in Y_{1}^{*}, y_{2} \in Y_{2}^{*}$ such that $\mu_{1}^{*}\left(q_{1}, x_{1}, p_{1}, y_{1}\right) \wedge \mu_{2}^{*}\left(q_{2}, x_{2}, p_{2}, y_{2}\right)=$

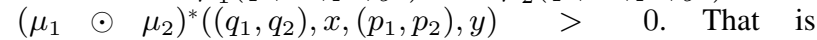
$\exists x_{1} \in X_{1}^{*}, x_{2} \in X_{2}^{*}$ and $\exists y_{1} \in Y_{1}^{*}, y_{2} \in Y_{2}^{*}$ such that $\mu_{1}^{*}\left(q_{1}, x_{1}, p_{1}, y_{1}\right)>0$ and $\mu_{2}^{*}\left(q_{2}, x_{2}, p_{2}, y_{2}\right)>0$. That is $p_{1} \in S\left(q_{1}\right)$ and $p_{2} \in S\left(q_{2}\right)$. That is $Q_{1}=S\left(q_{1}\right)$ and $Q_{2}=S\left(q_{2}\right)$. Thus $M_{1}$ and $M_{2}$ are cyclic.

2)When $\odot$ is full direct product. Suppose $M_{1}$ and $M_{2}$ are cyclic, say $Q_{1}=S\left(q_{1}\right)$ and $Q_{2}=S\left(q_{2}\right)$ for some $q_{1} \in Q_{1}$ and $q_{2} \in Q_{2}$. Let $\left(p_{1}, p_{2}\right) \in Q_{1} \times Q_{2}$. Then $\mu_{1}^{*}\left(q_{1}, x_{1}, p_{1}, y_{1}\right)>0$ and $\mu_{2}^{*}\left(q_{2}, x_{2}, p_{2}, y_{2}\right)>0$. Thus $\left(\mu_{1} \odot \mu_{2}\right)^{*}\left(\left(q_{1}, q_{2}\right),\left(x_{1}, x_{2}\right),\left(p_{1}, p_{2}\right),\left(y_{1}, y_{2}\right)\right)=$ $\mu_{1}^{*}\left(q_{1}, x_{1}, p_{1}, y_{1}\right) \wedge \mu_{2}^{*}\left(q_{2}, x_{2}, p_{2}, y_{2}\right)>0$. That is, $\left(p_{1}, p_{2}\right) \in S\left(\left(q_{1}, q_{2}\right)\right)$. Thus, $Q_{1} \odot Q_{2}=S\left(q_{1}, q_{2}\right)$.Hence $M_{1} \odot M_{2}$ is cyclic.

Conversely, Suppose $M_{1} \odot M_{2}$ is cyclic. Let $Q_{1} \odot Q_{2}=$ $S\left(\left(q_{1}, q_{2}\right)\right)$, for some $\left(q_{1}, q_{2}\right) \in Q_{1} \odot Q_{2}$. Let $p_{1} \in Q_{1}$ and $p_{2} \in$ $Q_{2}$. Then $\left(\mu_{1} \odot \mu_{2}\right)^{*}\left(\left(q_{1}, q_{2}\right),\left(x_{1}, x_{2}\right),\left(p_{1}, p_{2}\right),\left(y_{1}, y_{2}\right)\right)=$ $\mu_{1}^{*}\left(q_{1}, x_{1}, p_{1}, y_{1}\right) \wedge \mu_{2}^{*}\left(q_{2}, x_{2}, p_{2}, y_{2}\right)>0$. This implies $\mu_{1}^{*}\left(q_{1}, x_{1}, p_{1}, y_{1}\right)>0$ and $\mu_{2}^{*}\left(q_{2}, x_{2}, p_{2}, y_{2}\right)>0$. Therefore, $p_{1} \in S\left(q_{1}\right)$ and $p_{2} \in S\left(q_{2}\right)$.That is $Q_{1}=S\left(q_{1}\right)$, for some $q_{1} \in Q_{1}$ and $Q_{2}=S\left(q_{2}\right)$, for some $q_{2} \in Q_{2}$. Hence, $M_{1}$ and $M_{2}$ are cyclic.

Theorem 3.6 Let $M_{i}=\left(Q_{i}, X_{i}, Y_{i}, \mu_{i}\right)$ be a fmms, $i=1,2$ If fmms $M_{1} \odot M_{2}$ is cyclic then $M_{1}$ and $M_{2}$ are cyclic, where $\odot$ is restricted direct product, cascade product and wreath product. Proof. 1) When $\odot$ is restricted direct product. Suppose $M_{1} \odot M_{2}$ is cyclic. Let $Q_{1} \times Q_{2}=S\left(\left(q_{1}, q_{2}\right)\right)$, for some $\left(q_{1}, q_{2}\right) \in Q_{1} \times Q_{2}$. Let $p_{1} \in Q_{1}$ and $p_{2} \in Q_{2}$. Then $\left(\mu_{1} \odot \mu_{2}\right)^{*}\left(\left(q_{1}, q_{2}\right), x,\left(p_{1}, p_{2}\right), y\right)=$ $\mu_{1}^{*}\left(q_{1}, x, p_{1}, y\right) \odot \mu_{2}^{*}\left(q_{2}, x, p_{2}, y\right)>0$. This implies $\mu_{1}^{*}\left(q_{1}, x, p_{1}, y\right)>0$ and $\mu_{2}^{*}\left(q_{2}, x, p_{2}, y\right)>0$. Therefore, $p_{1} \in S\left(q_{1}\right)$ and $p_{2} \in S\left(q_{2}\right)$. That is $Q_{1}=S\left(q_{1}\right)$, for some $q_{1} \in Q_{1}$ and $Q_{2}=S\left(q_{2}\right)$, for some $q_{2} \in Q_{2}$. Hence, $M_{1}$ and $M_{2}$ are cyclic.

2) When $\odot$ is cascade product. Suppose $M_{1} \odot M_{2}$ is cyclic. Let $Q_{1} \times Q_{2}=S\left(\left(q_{1}, q_{2}\right)\right)$, for some $\left(q_{1}, q_{2}\right) \in Q_{1} \times Q_{2}$. Let $p_{1} \in$ 
$Q_{1}$ and $p_{2} \in Q_{2}$. Then $\left(\mu_{1} \odot \mu_{2}\right)^{*}\left(\left(q_{1}, q_{2}\right), x_{2},\left(p_{1}, p_{2}\right), y_{2}\right)=$ $\mu_{1}^{*}\left(q_{1}, \omega_{x}\left(q_{2}, x_{2}\right), p_{1}, \omega_{y}\left(q_{2}, y_{2}\right)\right) \wedge \mu_{2}^{*}\left(q_{2}, x_{2}, p_{2}, y_{2}\right)>0$. This implies $\mu_{1}^{*}\left(q_{1}, \omega_{x}\left(q_{2}, x_{2}\right), p_{1}, \omega_{y}\left(q_{2}, y_{2}\right)\right)>0$ and $\mu_{2}^{*}\left(q_{2}, x_{2}, p_{2}, y_{2}\right)>0$. Therefore, $p_{1} \in S\left(q_{1}\right)$ and $p_{2} \in S\left(q_{2}\right)$.That is $Q_{1}=S\left(q_{1}\right)$, for some $q_{1} \in Q_{1}$ and $Q_{2}=S\left(q_{2}\right)$, for some $q_{2} \in Q_{2}$. Hence, $M_{1}$ and $M_{2}$ are cyclic. 3) When $\odot$ is wreath product. Suppose $M_{1} \odot M_{2}$ is cyclic. Let $Q_{1} \times Q_{2}=S\left(\left(q_{1}, q_{2}\right)\right)$, for some $\left(q_{1}, q_{2}\right) \in Q_{1} \times Q_{2}$. Let $p_{1} \in$ $Q_{1}$ and $p_{2} \in Q_{2}$. Then $\left(\mu_{1} \odot \mu_{2}\right)^{*}\left(\left(q_{1}, q_{2}\right),\left(f, x_{2}\right),\left(p_{1}, p_{2}\right),(g\right.$ ,$\left.\left.y_{2}\right)\right)=\mu_{1}^{*}\left(q_{1}, f\left(q_{2}\right), p_{1}, g\left(q_{2}\right)\right) \wedge \mu_{2}^{*}\left(q_{2}, x_{2}, p_{2}, y_{2}\right)>0$. This implies $\mu_{1}^{*}\left(q_{1}, f\left(q_{2}\right), p_{1}, g\left(q_{2}\right)\right)>0$ and $\mu_{2}^{*}\left(q_{2}, x_{2}, p_{2}, y_{2}\right)>0$. Therefore, $p_{1} \in S\left(q_{1}\right)$ and $p_{2} \in S\left(q_{2}\right)$.That is $Q_{1}=S\left(q_{1}\right)$, for some $q_{1} \in Q_{1}$ and $Q_{2}=S\left(q_{2}\right)$, for some $q_{2} \in Q_{2}$. Hence, $M_{1}$ and $M_{2}$ are cyclic.

The converse of the above theorem is true when individual fmms are strongly connected.

Theorem 3.7 Let $M_{i}=\left(Q_{i}, X_{i}, Y_{i}, \mu_{i}\right)$ be a strongly connected fmms, $i=1,2$. Then $M_{1} \odot M_{2}$ is cyclic, where $\odot$ is restricted direct product, cascade product and wreath product.

Proof. 1) When $\odot$ is restricted direct product. By theorem (3.1) $M_{1}$ and $M_{2}$ are cyclic, one has $Q_{1}=S\left(q_{1}\right)$ and $Q_{2}=S\left(q_{2}\right)$ for some $q_{1} \in Q_{1}$ and $q_{2} \in Q_{2}$. Let $\left(p_{1}, p_{2}\right) \in Q_{1} \times Q_{2}$. Then $\mu_{1}^{*}\left(q_{1}, x, p_{1}, y\right)>0$ and $\mu_{2}^{*}\left(q_{2}, x, p_{2}, y\right)>0$. Thus $\left(\mu_{1} \odot \mu_{2}\right)^{*}\left(\left(q_{1}, q_{2}\right), x,\left(p_{1}, p_{2}\right), y\right)=$ $\mu_{1}^{*}\left(q_{1}, x, p_{1}, y\right) \wedge \mu_{2}^{*}\left(q_{2}, x, p_{2}, y\right)>0$. That is, $\left(p_{1}, p_{2}\right) \in S\left(\left(q_{1}, q_{2}\right)\right)$. Thus, $Q_{1} \times Q_{2}=S\left(q_{1}, q_{2}\right)$.Hence $M_{1} \odot M_{2}$ is cyclic.

2) When $\odot$ is cascade product. Since $M_{1}$ and $M_{2}$ are cyclic, one has $Q_{1}=S\left(q_{1}\right)$ and $Q_{2}=S\left(q_{2}\right)$ for some $q_{1} \in Q_{1}$ and $q_{2} \in$ $Q_{2}$. Let $\left(p_{1}, p_{2}\right) \in Q_{1} \times Q_{2}$. Then $\mu_{1}^{*}\left(q_{1}, \omega_{x}\left(q_{2}, x_{2}\right), p_{1}, \omega_{y}\right.$ $\left.\left(q_{2}, y_{2}\right)\right)>0$ and $\mu_{2}^{*}\left(q_{2}, x_{2}, p_{2}, y_{2}\right)>0$. Thus $\left(\mu_{1} \odot \mu_{2}\right)^{*}\left(\left(q_{1}, q_{2}\right), x_{2},\left(p_{1}, p_{2}\right), y_{2}\right)=\mu_{1}^{*}\left(q_{1}, \omega_{x}\right.$ $\left.\left(q_{2}, x_{2}\right), p_{1}, \omega_{y}\left(q_{2}, y_{2}\right)\right) \wedge \mu_{2}^{*}\left(q_{2}, x_{2}, p_{2}, y_{2}\right)>0$. That is, $\left(p_{1}, p_{2}\right) \in S\left(\left(q_{1}, q_{2}\right)\right)$. Thus, $Q_{1} \times Q_{2}=S\left(q_{1}, q_{2}\right)$.Hence $M_{1} \odot M_{2}$ is cyclic.

3) When $\odot$ is wreath product. Since $M_{1}$ and $M_{2}$ are cyclic, one has $Q_{1}=S\left(q_{1}\right)$ and $Q_{2}=S\left(q_{2}\right)$ for some $q_{1} \in Q_{1}$ and $q_{2} \in Q_{2}$. Let $\left(p_{1}, p_{2}\right) \in Q_{1} \times Q_{2}$. Then $\mu_{1}^{*}\left(q_{1}, f\left(q_{2}\right), p_{1}, g\left(q_{2}\right)\right)>0$ and $\mu_{2}^{*}\left(q_{2}, x_{2}, p_{2}, y_{2}\right)>0$. Thus $\quad\left(\mu_{1} \odot \mu_{2}\right)^{*}\left(\left(q_{1}, q_{2}\right),\left(f, x_{2}\right),\left(p_{1}, p_{2}\right),\left(g, y_{2}\right)\right)=$ $\mu_{1}^{*}\left(q_{1}, f\left(q_{2}\right), p_{1}, g\left(q_{2}\right)\right) \wedge \mu_{2}^{*}\left(q_{2}, x_{2}, p_{2}, y_{2}\right)>0$. That is, $\left(p_{1}, p_{2}\right) \in S\left(\left(q_{1}, q_{2}\right)\right)$. Thus, $Q_{1} \times Q_{2}=S\left(q_{1}, q_{2}\right)$. Hence $M_{1} \odot M_{2}$ is cyclic.

Theorem 3.8 Let $M_{i}=\left(Q_{i}, X_{i}, Y_{i}, \mu_{i}\right)$ be a fmms, $i=1,2$. Then fmms $M_{1} \odot M_{2}$ is retrievable if and only if $M_{1}$ and $M_{2}$ are retrievable, where $\odot$ is cartesian product and full direct product.

Proof. 1) When $\odot$ is cartesian product.Suppose $M_{1}$ and $M_{2}$ are retrievable. Let $\left(q_{1}, q_{2}\right),\left(p_{1}, p_{2}\right) \in Q_{1} \times Q_{2}$ and $x \in\left(X_{1} \cup X_{2}\right)^{*}, y \in\left(Y_{1} \cup Y_{2}\right)^{*}$ be such that $\left(\mu_{1} \odot \mu_{2}\right)^{*}\left(\left(q_{1}, q_{2}\right), x,\left(p_{1}, p_{2}\right), y\right)>0$. Let $x_{1}^{*}=x_{1} x_{2}$ be the standard form of $x, x_{1} \in X_{1}$ and $x_{2} \in X_{2}$ and $y_{1}^{*}=y_{1} y_{2}$ be the standard form of $y, y_{1} \in Y_{1}$ and $y_{2} \in Y_{2}$. Then $\left(\mu_{1} \odot \mu_{2}\right)^{*}\left(\left(q_{1}, q_{2}\right), x,\left(p_{1}, p_{2}\right), y\right)=\left(\mu_{1} \odot\right.$ $\left.\mu_{2}\right)^{*}\left(\left(q_{1}, q_{2}\right), x_{1} x_{2},\left(p_{1}, p_{2}\right), y_{1} y_{2}\right)=\mu_{1}^{*}\left(q_{1}, x_{1}, p_{1}, y_{1}\right) \wedge$ $\mu_{2}^{*}\left(q_{2}, x_{2}, p_{2}, y_{2}\right)$. Thus $\mu_{1}^{*}\left(q_{1}, x_{1}, p_{1}, y_{1}\right)>0 \quad$ and $\mu_{2}^{*}\left(q_{2}, x_{2}, p_{2}, y_{2}\right)>0$. Since $M_{1}$ and $M_{2}$ are retrievable, $\exists u_{1} \in X_{1}^{*}, u_{2} \in X_{2}^{*}, v_{1} \in Y_{1}^{*}, v_{2} \in Y_{2}^{*}$ such that $\mu_{1}^{*}\left(p_{1}, u_{1}, q_{1}, v_{1}\right)>0$ and $\mu_{2}^{*}\left(p_{2}, u_{2}, q_{2}, v_{2}\right)>0$. Thus $\left(\mu_{1} \odot \mu_{2}\right)^{*}\left(\left(p_{1}, p_{2}\right), u_{1} u_{2},\left(q_{1}, q_{2}\right), v_{1} v_{2}\right)>0$. That is $\left(\mu_{1} \odot \mu_{2}\right)^{*}\left(\left(p_{1}, p_{2}\right), u,\left(q_{1}, q_{2}\right), v\right)>0$. Hence, $M_{1} \odot M_{2}$ is retrievable.

Conversely, suppose $M_{1} \odot M_{2}$ is retrievable. Let $q_{1}, p_{1} \in Q_{1}$, $x \in X_{1}^{*}$ and $y \in Y_{1}^{*}$ be such that $\mu_{1}^{*}\left(q_{1}, x_{1}, p_{1}, y_{1}\right)>0$. Then $\forall q_{2} \in Q_{2},\left(\mu_{1} \odot \mu_{2}\right)^{*}\left(\left(q_{1}, q_{2}\right), x_{1},\left(p_{1}, q_{2}\right), y_{1}\right)>0$ Thus $\exists u \in\left(X_{1} \cup X_{2}\right)^{*}$ and $\exists v \in\left(Y_{1} \cup Y_{2}\right)^{*}$ such that $\left(\mu_{1} \odot \mu_{2}\right)^{*}\left(\left(q_{1}, q_{2}\right), u,\left(p_{1}, q_{2}\right), v\right)>0$. Let $u=u_{1} u_{2}$ be the standard form of $u$ and $v$ respectively where $u_{1} \in X_{1}^{*}, u_{2} \in X_{2}^{*}$ and $v_{1} \in Y_{1}^{*}, v_{2} \in Y_{2}^{*}$. Then $0<$ $\left(\mu_{1} \odot \mu_{2}\right)^{*}\left(\left(q_{1}, q_{2}\right), u_{1} u_{2},\left(p_{1}, q_{2}\right), v_{1} v_{2}\right)=\mu_{1}\left(q_{1}, u_{1}, p_{1}, v_{1}\right) \wedge$ $\mu_{2}\left(q_{2}, u_{2}, q_{2}, v_{2}\right)$. Thus $\mu_{1}\left(q_{1}, u_{1}, p_{1}, v_{1}\right)>0$. Hence $M_{1}$ is retrievable. Similarly $M_{2}$ is retrievable.

2)When $\odot$ is full direct product. Suppose $M_{1}$ and $M_{2}$ are retrievable. Let $\left(q_{1}, q_{2}\right),\left(p_{1}, p_{2}\right) \in Q_{1} \times Q_{2}$ and $\left(x_{1}, x_{2}\right) \in\left(X_{1} \times X_{2}\right)^{*},\left(y_{1}, y_{2}\right) \in\left(Y_{1} \times Y_{2}\right)^{*}$ be such that $\left(\mu_{1} \odot \mu_{2}\right)^{*}\left(\left(q_{1}, q_{2}\right),\left(x_{1}, x_{2}\right), \quad\left(p_{1}, p_{2}\right),\left(y_{1}, y_{2}\right)\right)=$ $\mu_{1}^{*}\left(q_{1}, x_{1}, p_{1}, y_{1}\right) \wedge \mu_{2}^{*}\left(q_{2}, x_{2}, p_{2}, y_{2}\right)>0$. Since, $M_{1}$ and $M_{2}$ are retrievable. $\exists u_{1} \in X_{1}^{*}, v_{1} \in Y_{1}^{*}$ and $\exists u_{2} \in X_{2}^{*}, v_{2} \in Y_{2}^{*}$, such that $\mu_{1}^{*}\left(p_{1}, u_{1}, q_{1}, v_{1}\right)>0$ and $\mu_{2}^{*}\left(p_{2}, u_{2}, q_{2}, v_{2}\right)>0$. Now $\mu_{1}^{*}\left(p_{1}, u_{1}, q_{1}, v_{1}\right) \wedge$ $\mu_{2}^{*}\left(p_{2}, u_{2}, q_{2}, v_{2}\right)=\left(\mu_{1} \times \mu_{2}\right)^{*}\left(\left(p_{1}, p_{2}\right),\left(u_{1}, u_{2}\right)\right.$, $\left.\left(q_{1}, q_{2}\right),\left(v_{1}, v_{2}\right)\right)>0$. Therefore, $M_{1} \odot M_{2}$ is retrievable.

Conversely, suppose $M_{1} \odot M_{2}$ is retrievable. Let $\left(q_{1}, q_{2}\right) \in Q_{1} \times Q_{2},\left(x_{1}, x_{2}\right) \in\left(X_{1} \times X_{2}\right)^{*}$ and $\left(y_{1}, y_{2}\right) \in\left(Y_{1} \times Y_{2}\right)^{*} \exists\left(p_{1}, p_{2}\right) \in Q_{1} \times Q_{2}$ such that $\left(\mu_{1} \odot \mu_{2}\right)^{*}\left(\left(q_{1}, q_{2}\right),\left(x_{1}, x_{2}\right),\left(p_{1}, p_{2}\right),\left(y_{1}, y_{2}\right)\right)>0$ then $\exists\left(u_{1}, u_{2}\right) \in\left(X_{1} \times X_{2}\right)^{*},\left(v_{1}, v_{2}\right) \in\left(Y_{1} \times Y_{2}\right)^{*}$ such that $\left(\mu_{1} \odot \mu_{2}\right)^{*}\left(\left(p_{1}, p_{2}\right), \quad\left(u_{1}, u_{2}\right),\left(q_{1}, q_{2}\right),\left(v_{1}, v_{2}\right)\right)=$ $\mu_{1}^{*}\left(p_{1}, u_{1}, q_{1}, v_{1}\right) \wedge \mu_{2}^{*}\left(p_{2}, u_{2}, q_{2}, v_{2}\right)>0$. Hence, $M_{1}$ and $M_{2}$ are retrievable.

Theorem 3.9 Let $M_{i}=\left(Q_{i}, X_{i}, Y_{i}, \mu_{i}\right)$ be a fmms, $i=1,2$ If fmms $M_{1} \odot M_{2}$ is retrievable then $M_{1}$ and $M_{2}$ are retrievable, where $\odot$ is restricted direct product, cascade product and wreath product.

Proof. 1) When $\odot$ is restricted direct product. Suppose $M_{1} \odot M_{2}$ is retrievable. Let $\left(q_{1}, q_{2}\right),\left(p_{1}, p_{2}\right) \in Q_{1} \times Q_{2}, x \in X$ and $y \in Y$ such that $\left(\mu_{1} \times \mu_{2}\right)^{*}\left(\left(q_{1}, q_{2}\right), x,\left(p_{1}\right.\right.$, $\left.\left.p_{2}\right), y\right)>0$ then $\exists u \in X^{*}, v \in Y^{*}$ such that $\left(\mu_{1} \times \mu_{2}\right)^{*}\left(\left(p_{1}, p_{2}\right), u,\left(q_{1}, q_{2}\right), v\right)=\mu_{1}^{*}\left(p_{1}, u, q_{1}, v\right)$ $\wedge \mu_{2}^{*}\left(p_{2}, u, q_{2}, v\right)>0$. Hence, $M_{1}$ and $M_{2}$ are retrievable.

2) When $\odot$ is cascade product. Suppose $M_{1} \odot M_{2}$ is retrievable. Let $\left(q_{1}, q_{2}\right),\left(p_{1}, p_{2}\right) \in Q_{1} \times Q_{2}, x_{2} \in X_{2}$ and $y_{2} \in Y_{2}$ such that $\left(\mu_{1} \odot \mu_{2}\right)^{*}\left(\left(q_{1}, q_{2}\right), x_{2},\left(p_{1}, p_{2}\right), y_{2}\right)>0$ then $\exists u_{2} \in$ $X_{2}^{*}, v_{2} \in Y_{2}^{*}$ such that $\left(\mu_{1} \odot \mu_{2}\right)^{*}\left(\left(p_{1}, p_{2}\right), u_{2},\left(q_{1}, q_{2}\right), v_{2}\right)=$ $\mu_{1}^{*}\left(p_{1}, \omega_{u}\left(p_{2}, u_{2}\right), q_{1}, \omega_{v}\left(p_{2}, v_{2}\right)\right) \wedge \mu_{2}^{*}\left(p_{2}, u_{2}, q_{2}, v_{2}\right)>0$. Hence, $M_{1}$ and $M_{2}$ are retrievable.

3) When $\odot$ is wreath product. Suppose $M_{1} \odot M_{2}$ is retrievable. Let $\left(q_{1}, q_{2}\right),\left(p_{1}, p_{2}\right) \in Q_{1} \times Q_{2}, x_{2} \in X_{2}^{*}$ and $y_{2} \in Y_{2}^{*}$ such that $\left(\mu_{1} \odot \mu_{2}\right)^{*}\left(\left(q_{1}, q_{2}\right),\left(f, x_{2}\right),\left(p_{1}, p_{2}\right),\left(g, y_{2}\right)\right)>0$ then $\exists u_{2} \in X_{2}^{*}, v_{2} \in Y_{2}^{*}$ such that $\left(\mu_{1} \odot\right.$ $\left.\mu_{2}\right)^{*}\left(\left(p_{1}, p_{2}\right),\left(f, u_{2}\right),\left(q_{1}, q_{2}\right),\left(g, v_{2}\right)\right)=\mu_{1}^{*}\left(p_{1}, f\left(p_{2}\right), q_{1}\right.$, $\left.g\left(p_{2}\right)\right) \wedge \mu_{2}^{*}\left(p_{2}, u_{2}, q_{2}, v_{2}\right)>0$. Hence, $M_{1}$ and $M_{2}$ are retrievable.

The converse of the above theorem is true when individual fmms are strongly connected.

Theorem 3.10 Let $M_{i}=\left(Q_{i}, X_{i}, Y_{i}, \mu_{i}\right)$ be a strongly connected fmms, $i=1,2$. Then $M_{1} \odot M_{2}$ is retrievable, where $\odot$ is restricted direct product, cascade product and wreath product. Proof. 1) When $\odot$ is restricted direct product. By theorem (3.1) $M_{1}$ and $M_{2}$ are retrievable. Let $\left(q_{1}, q_{2}\right),\left(p_{1}, p_{2}\right) \in Q_{1} \times Q_{2}$ and $x \in X^{*}, y \in Y^{*}$ be such that $\left(\mu_{1} \odot \mu_{2}\right)^{*}\left(\left(q_{1}, q_{2}\right), x,\left(p_{1}, p_{2}\right), y\right)=$ $\mu_{1}^{*}\left(q_{1}, x, p_{1}, y\right) \wedge \mu_{2}^{*}\left(q_{2}, x, p_{2}, y\right)>0$. Since, $M_{1}$ and $M_{2}$ are retrievable. $\exists u \in X^{*}, v \in Y^{*}$, such that $\mu_{1}^{*}\left(p_{1}, u, q_{1}, v\right)>0$ and $\mu_{2}^{*}\left(p_{2}, u, q_{2}, v\right)>0$. Now $\mu_{1}^{*}\left(p_{1}, u, q_{1}, v\right) \wedge \mu_{2}^{*}\left(p_{2}, u, q_{2}, v\right)=$ $\left(\mu_{1} \odot \mu_{2}\right)^{*}\left(\left(p_{1}, p_{2}\right), u,\left(q_{1}, q_{2}\right), v\right)>0$. Therefore, $M_{1} \odot M_{2}$ is retrievable.

2) When $\odot$ is cascade product. Suppose $M_{1}$ and $M_{2}$ are retrievable. Let $\left(q_{1}, q_{2}\right),\left(p_{1}, p_{2}\right) \in Q_{1} \times Q_{2}$ and $x_{2} \in X_{2}^{*}, y_{2} \in Y_{2}^{*}$ be such that $\left(\mu_{1} \odot \mu_{2}\right)^{*}\left(\left(q_{1}, q_{2}\right), x_{2},\left(p_{1}, p_{2}\right), y_{2}\right)=\mu_{1}^{*}\left(q_{1}, \omega_{x}\left(q_{2}\right.\right.$, $\left.\left.x_{2}\right), p_{1}, \omega_{y}\left(q_{2}, y_{2}\right)\right) \wedge \mu_{2}^{*}\left(q_{2}, x_{2}, p_{2}, y_{2}\right)>0$. Since, $M_{1}$ and $M_{2}$ are retrievable. $\exists u_{2} \in X_{2}^{*}, v_{2} \in Y_{2}^{*}$, such that $\mu_{1}^{*}\left(p_{1}, \omega_{u}\left(q_{2}, u_{2}\right), q_{1}, \omega_{v}\left(q_{2}, v_{2}\right)\right)>0$ and $\mu_{2}^{*}\left(p_{2}, u_{2}, q_{2}, v_{2}\right)>0$. Now $\mu_{1}^{*}\left(p_{1}, \odot_{u}\left(q_{2}, u_{2}\right)\right.$, $\left.q_{1}, \odot_{v}\left(q_{2}, v_{2}\right)\right) \wedge \mu_{2}^{*}\left(p_{2}, u_{2}, q_{2}, v_{2}\right) \rightleftharpoons\left(\mu_{1} \odot\right.$ $\left.\mu_{2}\right)^{*}\left(\left(p_{1}, p_{2}\right), u_{2},\left(q_{1}, q_{2}\right), v_{2}\right)>0$. Therefore, $M_{1} \odot M_{2}$ 
is retrievable.

3) When $\odot$ is wreath product. Suppose $M_{1}$ and $M_{2}$ are retrievable. Let $\left(q_{1}, q_{2}\right),\left(p_{1}, p_{2}\right) \in Q_{1} \times Q_{2}$ and $x_{2} \in X_{2}^{*}, y_{2} \in Y_{2}^{*}$ be such that $\left(\mu_{1} \odot \mu_{2}\right)^{*}\left(\left(q_{1}, q_{2}\right),\left(f, x_{2}\right),\left(p_{1}, p_{2}\right),\left(g, y_{2}\right)\right)=$ $\mu_{1}^{*}\left(p_{1}, f\left(p_{2}\right), q_{1}, g\left(p_{2}\right)\right) \wedge \mu_{2}^{*}\left(q_{2}, x_{2}, p_{2}, y_{2}\right)>0$. Since, $M_{1}$ and $M_{2}$ are retrievable. $\exists u_{2} \in X_{2}^{*}, v_{2} \in Y_{2}^{*}$, such that $\mu_{1}^{*}\left(p_{1}, f\left(p_{2}\right)\right.$, $\left.q_{1}, g\left(p_{2}\right)\right)>0$ and $\mu_{2}^{*}\left(p_{2}, u_{2}, q_{2}, v_{2}\right)>0$. Now $\mu_{1}^{*}\left(p_{1}, f\left(p_{2}\right)\right.$, $\left.q_{1}, g\left(p_{2}\right)\right) \wedge \mu_{2}^{*}\left(p_{2}, u_{2}, q_{2}, v_{2}\right)=\left(\mu_{1} \odot \mu_{2}\right)^{*}\left(\left(p_{1}, p_{2}\right),(g\right.$, $\left.\left.u_{2}\right),\left(q_{1}, q_{2}\right),\left(g, v_{2}\right)\right)>0$. Therefore, $M_{1} \odot M_{2}$ is retrievable.

Remark 3.2 Let $M_{i}=\left(Q_{i}, X_{i}, Y_{i}, \mu_{i}\right)$ be a fmms, then by theorem (2.7),(3.8) and (3.9)

(1) Fmm $M_{1} \odot M_{2}$ is union of strongly connected submachines (exchange property) if and only if $M_{1}$ and $M_{2}$ are union of strongly connected submachines (exchange property), where $\odot$ is cartesian product and full direct product.

(2) Fmm $M_{1} \odot M_{2}$ is union of strongly connected submachines(exchange property) then $M_{1}$ and $M_{2}$ are union of strongly connected submachines (exchange property), where $\odot$ is restricted direct product, cascade product and wreath product.

Remark 3.3 Let $M_{i}=\left(Q_{i}, X_{i}, Y_{i}, \mu_{i}\right)$ be a strongly connected fmms, then by theorem (2.7),(3.5) and (3.10) $M_{1} \odot M_{2}$ is union of strongly connected submachines (exchange property), where $\odot$ is restricted direct product, cascade product and wreath product.

Theorem 3.11 Let $M_{i}=\left(Q_{i}, X_{i}, Y_{i}, \mu_{i}\right)$ be a fmms, $i=1,2$. Then fmm $M_{1} \odot M_{2}$ is connected if and only if $M_{1}$ and $M_{2}$ are connected, where $\odot$ is cartesian product and full direct product. Proof. 1) When $\odot$ is cartesian product.

Suppose $M_{1}$ and $M_{2}$ are connected. Let $\left(q_{1}, q_{2}\right),\left(p_{1}, p_{2}\right) \in$ $Q_{1} \times Q_{2}$, Now $\exists q_{10}, q_{11}, \ldots, q_{1 n} \in Q_{1}, q_{1}=q_{10}, p_{1}=q_{1 n}$ and $\exists a_{11}, a_{12}, \ldots, a_{1 n} \in X_{1}$ and $\exists b_{11}, b_{12}, \ldots, b_{1 n} \in Y_{1}$ $\forall i=1,2, \ldots, n$ either $\mu_{1}\left(q_{1 i-1}, a_{1 i}, q_{1 i}, b_{1 i}\right)>0$ or $\mu_{1}\left(q_{1 i}, a_{1 i}, q_{1 i-1}, b_{1 i}\right)>0$ and $\exists q_{20}, q_{21}, \ldots, q_{2 m} \in Q_{2}$, $q_{2}=q_{20}, p_{2}=q_{2 m}$ and $\exists a_{21}, a_{22}, \ldots, a_{2 m} \in X_{2}$ and $\exists b_{21}, b_{22}, \ldots, b_{2 m} \in Y_{2} \forall i=1,2, \ldots, m$ either $\mu_{2}\left(q_{2 i-1}, a_{2 i}, q_{2 i}, b_{2 i}\right)>0$ or $\mu_{2}\left(q_{2 i}, a_{2 i}, q_{2 i-1}, b_{2 i}\right)>$ 0 . Consider the sequence of states $\left(q_{1}, q_{2}\right)=$ $\left(q_{10}, q_{20}\right),\left(q_{11}, q_{20}\right), \ldots,\left(q_{1 n}, q_{20}\right),\left(q_{1 n}, q_{21}\right), \ldots\left(q_{1 n}, q_{2 m}\right)=$ $\left(p_{1}, p_{2}\right) \in Q_{1} \times Q_{2}$ and the sequence $a_{11}, a_{12}, \ldots, a_{1 n}, a_{21}, a_{22}, \ldots, a_{2 m} \in X_{1} \cup X_{2}$ and $b_{11}, b_{12}, \ldots, b_{1 n}, b_{21}, b_{22}, \ldots, b_{2 m} \in Y_{1} \cup Y_{2} . \forall i=1,2, \ldots, n$ either $\left.\mu_{1} \odot \mu_{2}\left(\left(q_{1 i-1}, q_{20}\right), a_{1 i},\left(q_{1 i}, q_{20}\right), b_{1 i}\right)\right)>0$ or $\left.\mu_{1} \odot \mu_{2}\left(\left(q_{1 i}, q_{20}\right), a_{1 i},\left(q_{1 i-1}, q_{20}\right), b_{1 i}\right)\right)>0$ and $\forall j=1,2, \ldots, m$ either $\mu_{1} \odot \mu_{2}\left(\left(q_{1 n}, q_{2 j-1}\right), a_{2 j},\left(q_{1 n}\right.\right.$, $\left.\left.\left.q_{2 j}\right), b_{2 j}\right)\right)>0$ or $\left.\mu_{1} \odot \mu_{2}\left(\left(q_{1 n}, q_{2 j}\right), a_{2 j},\left(q_{1 n}, q_{2 j-1}\right), b_{2 j}\right)\right)>$ 0 . Hence $\left(q_{1}, q_{2}\right)$ and $\left(p_{1}, p_{2}\right)$ are connected. i.e. $M_{1} \odot M_{2}$ is connected

Conversely Suppose that $M_{1} \odot M_{2}$ is connected. Let $q_{1}, p_{1} \in Q_{1}$ and let $r_{2} \in Q_{2}$. If $p_{1}=q_{1}$ then $p_{1}$ and $q_{1}$ are connected. Suppose, $p_{1} \neq q_{1}$ Then $\exists\left(q_{1}, r_{2}\right)=$ $\left(q_{10}, r_{20}\right),\left(q_{11}, r_{21}\right), \ldots,\left(q_{1 n}, r_{2 n}\right)=\left(p_{1}, r_{2}\right) \in Q_{1} \times Q_{2}$ and $a_{1}, a_{2}, \ldots, a_{n} \in X_{1} \cup X_{2}$ such that $\forall i=1,2, \ldots, n$ either $\left.\mu_{1} \odot \mu_{2}\left(\left(q_{1 i-1}, r_{2 i-1}\right), a_{i},\left(q_{1 i}, r_{2 i}\right), b_{i}\right)\right)>0$ or $\left.\mu_{1} \odot \mu_{2}\left(\left(q_{1 i}, r_{2 i}\right), a_{i},\left(q_{1 i-1}, r_{2 i-1}\right), b_{i}\right)\right)>0$ Clearly, if $q_{1 i-1} \neq q_{1 i}$ then $r_{2 i-1}=r_{2 i}$ and if $r_{2 i-1} \neq r_{2 i}$ then $q_{1 i-1}=q_{1 i} \forall i=1,2, \ldots, n$ Let $\left\{q_{1}=q_{11}^{\prime}, q_{12}^{\prime}, q_{13}^{\prime}, \ldots, q_{1 k}^{\prime}=p_{1}\right\}$ be the set of all distinct $q_{1 i}^{\prime} \in\left\{q_{10}, q_{11}, \ldots, q_{1 n}\right\}$ and let $a_{1}^{\prime}, a_{2}^{\prime}, \ldots, a_{k}^{\prime} \in X_{1}$ and $b_{1}^{\prime}, b_{2}^{\prime}, \ldots, b_{k}^{\prime} \in Y_{1}$ be the corresponding $a_{i}$ 's and $b_{i}$ 's respectively and $\forall j=1,2, \ldots, k$ either $\mu_{1}\left(q_{1 j-1}^{\prime}, a_{j}^{\prime}, q_{1 j}^{\prime}, b_{j}^{\prime}\right)>0$ or $\mu_{1}\left(q_{1 j}^{\prime}, a_{j}^{\prime}, q_{1 j-1}^{\prime}, b_{j}^{\prime}\right)>0$ Thus $p_{1}$ and $q_{1}$ are connected and hence $M_{1}$ is connected. Similarly we can show that $M_{2}$ is connected.

2) When $\odot$ is full direct product.

case(i) Suppose $M_{1}$ and $M_{2}$ are connected. Let $\left(q_{1}, q_{2}\right),\left(p_{1}, p_{2}\right) \in Q_{1} \times Q_{2}$, Now $\exists q_{10}, q_{11}, \ldots$, $q_{1 n} \in Q_{1}, q_{1}=q_{10}, p_{1}=q_{1 n}$ and $\exists a_{11}, a_{12}, \ldots, a_{1 n} \in$ $X_{1}$ and $\exists b_{11}, b_{12}, \ldots, b_{1 n} \in Y_{1} \quad \forall i=1,2, \ldots, n$ $\mu_{1}\left(q_{1 i-1}, a_{1 i}, q_{1 i}, b_{1 i}\right)>0$ and $\exists q_{20}, q_{21}, \ldots, q_{2 m} \in Q_{2}$, $q_{2}=q_{20}, p_{2}=q_{2 m}$ and $\exists a_{21}, a_{22}, \ldots, a_{2 m} \in X_{2}$ and $\exists b_{21}, b_{22}, \ldots, b_{2 m} \in Y_{2} . \quad \forall i=1,2, \ldots, m$ $\mu_{2}\left(q_{2 i-1}, a_{2 i}, q_{2 i}, b_{2 i}\right)>0$ Without loss of generality $m \leq n$. Consider the sequence of states $\left(q_{1}, q_{2}\right)=\left(q_{10}, q_{20}\right),\left(q_{11}, q_{21}\right), \ldots,\left(q_{1 m}, q_{2 m}\right),\left(q_{1 m+1}\right.$, $\left.q_{2 m}\right), \ldots,\left(q_{1 n}, q_{2 m}\right)=\left(p_{1}, p_{2}\right)$ and a sequence $\left(a_{11}, a_{21}\right)$, $\ldots,\left(a_{1 m}, a_{2 m}\right), \quad\left(a_{1 m+1}, \quad a_{2 m+1}\right), \ldots,\left(a_{1 n}, a_{2 n}\right)$ where $a_{2 k}=\lambda, \forall k=m+1, \ldots, n$. and a sequence $\left(b_{11}, b_{21}\right), .$. $.,\left(b_{1 m}, b_{2 m}\right), \quad\left(b_{1 m+1}, \quad b_{2 m+1}\right), \ldots,\left(b_{1 n}, b_{2 n}\right) \quad$ where $b_{2 k}=\lambda, \forall k=m+1, \ldots, n$. Then $\forall i=1,2, \ldots, n, \mu_{1} \odot$ $\mu_{2}\left(\left(q_{1 i-1}, q_{2 i-1}\right),\left(a_{1 i}, a_{2 i}\right),\left(q_{1 i}, q_{2 i}\right),\left(b_{1 i}, b_{2 i}\right)\right)>0$, where $q_{2 i}=q_{2 m}, \forall i=m+1, \ldots, n$. Hence $\left(q_{1}, q_{2}\right)$ and $\left(p_{1}, p_{2}\right)$ are connected. i.e. $M_{1} \odot M_{2}$ is connected.

case(ii) Suppose $M_{1}$ and $M_{2}$ are connected. Let $\left(q_{1}, q_{2}\right),\left(p_{1}, p_{2}\right) \in Q_{1} \times Q_{2}$, Now $\exists q_{10}, q_{11}, \ldots$, $q_{1 n} \in Q_{1}, q_{1}=q_{10}, p_{1}=q_{1 n}$ and $\exists a_{11}, a_{12}, \ldots, a_{1 n} \in$ $X_{1}$ and $\exists b_{11}, b_{12}, \ldots, b_{1 n} \in Y_{1} \quad \forall i=1,2, \ldots, n$ $\mu_{1}\left(q_{1 i}, a_{1 i}, q_{1 i-1}, b_{1 i}\right)>0$ and $\exists q_{20}, q_{21}, \ldots, q_{2 m} \in Q_{2}$, $q_{2}=q_{20}, p_{2}=q_{2 m}$ and $\exists a_{21}, a_{22}, \ldots, a_{2 m} \in X_{2}$ and $\exists b_{21}, b_{22}, \ldots, b_{2 m} \in Y_{2} . \forall i=1,2, \ldots, m$ $\mu_{2}\left(q_{2 i}, a_{2 i}, q_{2 i-1}, b_{2 i}\right)>0$ Without loss of generality $m \leq n$. Consider the sequence of states $\left(p_{1}, p_{2}\right)=\left(q_{1 n}, q_{2 m}\right),\left(q_{1 n-1}, q_{2 m}\right), \ldots,\left(q_{1 m}, q_{2 m}\right)$, $\left(q_{1 m-1}, \quad q_{2 m-1}\right), \ldots,\left(q_{11}, q_{21}\right),\left(q_{10}, q_{20}\right)=\left(q_{1}, q_{2}\right)$ and a sequence $\left(a_{1 n}, a_{2 n}\right), \ldots,\left(a_{1 m}, a_{2 m}\right), \quad\left(a_{1 m-1}\right.$, $\left.a_{2 m-1}\right), \ldots,\left(a_{10}, a_{20}\right)$ where $a_{2 k}=\lambda, \forall k=m+1, \ldots, n$. and a sequence $\left(b_{1 n}, b_{2 n}\right), \ldots,\left(b_{1 m}, b_{2 m}\right), \quad\left(b_{1 m-1}\right.$, $\left.b_{2 m-1}\right), \ldots,\left(b_{10}, b_{20}\right)$ where $b_{2 k}=\lambda, \forall k=m+1, \ldots, n$. Then $\left.\forall i=n, n-1, \ldots, 1, \mu_{1} \odot \mu_{2}\left(\left(q_{1 i}, q_{2 i}\right), a_{1 i},\left(q_{1 i}, q_{2 i}\right), b_{1 i}\right)\right)>0$, where $q_{2 i}=q_{2 m}, \forall i=m+1, \ldots, n$.Hence $\left(q_{1}, q_{2}\right)$ and $\left(p_{1}, p_{2}\right)$ are connected. i.e. $M_{1} \odot M_{2}$ is connected.

case(iii) Suppose $M_{1}$ and $M_{2}$ are connected. Let $\left(q_{1}, q_{2}\right),\left(p_{1}, p_{2}\right) \in Q_{1} \times Q_{2}$, Now $\exists q_{10}, q_{11}, \ldots$, $q_{1 n} \in Q_{1}, q_{1}=q_{10}, p_{1}=q_{1 n}$ and $\exists a_{11}, a_{12}, \ldots, a_{1 n} \in$ $X_{1}$ and $\exists b_{11}, b_{12}, \ldots, b_{1 n} \in Y_{1} \quad \forall i=1,2, \ldots, n$ $\mu_{1}\left(q_{1 i-1}, a_{1 i}, q_{1 i}, b_{1 i}\right)>0$ and $\exists q_{20}, q_{21}, \ldots, q_{2 m} \in Q_{2}$, $q_{2}=q_{2 m}, p_{2}=q_{20}$ and $\exists a_{21}, a_{22}, \ldots, a_{2 m} \in X_{2}$ and $\exists b_{21}, b_{22}, \ldots, b_{2 m} \in Y_{2} \cdot \forall i \quad \forall i=1,2, \ldots, m$ $\mu_{2}\left(q_{2 i}, a_{2 i}, q_{2 i-1}, b_{2 i}\right)>0$ Without loss of generality $m \leq n$. Consider the sequence of states $\left(q_{1}, q_{2}\right)=\left(q_{10}, q_{2 m}\right),\left(q_{11}\right.$, $\left.q_{2 m-1}\right), \ldots,\left(q_{1 m}, q_{20}\right),\left(q_{1 m+1}, q_{20}\right), \ldots,\left(q_{1 n}, q_{20}\right)=\left(p_{1}, p_{2}\right)$ and a sequence $\left(a_{11}, a_{2 m}\right), \ldots,\left(a_{1 m}, a_{21}\right), \quad\left(a_{1 m+1}\right.$, $\left.a_{2 m+1}\right), \ldots,\left(a_{1 n}, a_{2 n}\right)$ where $a_{2 k}=\lambda, \forall k=m+1, \ldots, n$ and a sequence $\left(b_{11}, b_{2 m}\right), \quad \ldots,\left(b_{1 m}, b_{21}\right), \quad\left(b_{1 m+1}\right.$, $\left.b_{2 m+1}\right), \ldots,\left(b_{1 n}, b_{2 n}\right)$ where $b_{2 k}=\lambda \forall k=m+1, \ldots, n$.Hence $\left(q_{1}, q_{2}\right)$ and $\left(p_{1}, p_{2}\right)$ are connected. i.e. $M_{1} \odot M_{2}$ is connected. case(iv) Suppose $M_{1}$ and $M_{2}$ are connected. Let $\left(q_{1}, q_{2}\right),\left(p_{1}, p_{2}\right) \in Q_{1} \times Q_{2}$, Now $\exists q_{10}, q_{11}, \ldots$, $q_{1 n} \in Q_{1}, p_{1}=q_{10}, q_{1}=q_{1 n}$ and $\exists a_{11}, a_{12}, \ldots, a_{1 n} \in$ $X_{1}$ and $\exists b_{11}, b_{12}, \ldots, b_{1 n} \in Y_{1} \quad \forall i=1,2, \ldots, n$ $\mu_{1}\left(q_{1 i}, a_{1 i}, q_{1 i-1}, b_{1 i}\right)>0$ and $\exists q_{20}, q_{21}, \ldots, q_{2 m} \in Q_{2}$, $q_{2}=q_{20}, p_{2}=q_{2 m}$ and $\exists a_{21}, a_{22}, \ldots, a_{2 m} \in X_{2}$ and $\exists b_{21}, b_{22}, \ldots, b_{2 m} \in Y_{2} . \quad \forall i=1,2, \ldots, m$ $\mu_{2}\left(q_{2 i-1}, a_{2 i}, q_{2 i}, b_{2 i}\right)>0$ Without loss of generality $m \leq n$. Consider the sequence of states $\left(q_{1}, q_{2}\right)=\left(q_{1 n}, q_{20}\right),\left(q_{1 n-1}, q_{20}\right), \ldots,\left(q_{1 m+1}, q_{20}\right)\left(q_{1 m}, q_{20}\right)$, $\left(q_{1 m-1}, q_{21}\right), \ldots,\left(q_{10}, q_{2 m}\right)=\left(p_{1}, p_{2}\right)$ and a sequence $\left(a_{1 n}, a_{2 n}\right), \quad \ldots,\left(a_{1 m}, a_{21}\right), \quad\left(a_{1 m-1}, a_{22}\right), \ldots,\left(a_{1 n}, a_{2 m}\right)$ where $a_{2 k}=\lambda, \forall k=m+1, \ldots, n$ and a sequence $\left(b_{1 n}, b_{2 n}\right), \ldots,\left(b_{1 m}, b_{21}\right),\left(b_{1 m-1}, b_{22}\right), \ldots,\left(b_{1 n}, b_{2 m}\right)$ where $b_{2 k}=\lambda, \forall k=m+1, \ldots, n$.Hence $\left(q_{1}, q_{2}\right)$ and $\left(p_{1}, p_{2}\right)$ are connected. i.e. $M_{1} \odot M_{2}$ is connected.

Conversely Suppose $M_{1} \odot M_{2}$ is connected. Let $\left(q_{1}, q_{2}\right),\left(p_{1}, p_{2}\right) \in Q_{1} \times Q_{2}$, Now $\exists$ a sequence of states $\left\{\left(q_{1}, q_{2}\right)=\left(q_{10}, q_{20}\right),\left(q_{11}, q_{21}\right), \ldots,\left(q_{1 n}, q_{2 n}\right)=\right.$ $\left.\left(p_{1}, p_{2}\right)\right\} \in Q_{1} \times Q_{2}$ and the sequence 
$\left\{\left(a_{11}, a_{21}\right),\left(a_{12}, a_{22}\right), \ldots,\left(a_{1 n}, a_{2 n}\right)\right\} \in X_{1} \times X_{2}$ and $\left\{\left(b_{11}, b_{21}\right),\left(b_{12}, b_{22}\right), \ldots,\left(b_{1 n}, b_{2 n}\right)\right\} \in Y_{1} \times Y_{2}$. $\forall i=1,2, \ldots, n$ either $\mu_{1} \odot \mu_{2}\left(\left(q_{1 i-1}, q_{2 i-1}\right),\left(a_{1 i}, a_{2 i}\right)\right.$ $\left.,\left(q_{1 i}, q_{2 i}\right),\left(b_{1 i}, b_{2 i}\right)\right)>0$ or $\mu_{1} \odot \mu_{2}\left(\left(q_{1 i}, q_{2 i}\right),\left(a_{1 i}, a_{2 i}\right)\right.$ $\left.,\left(q_{1 i-1}, q_{2 i-1}\right),\left(b_{1 i}, b_{2 i}\right)\right)>0$. Without loss of generality, suppose $\mu_{1} \odot \mu_{2}\left(\left(q_{1 i-1}, q_{2 i-1}\right),\left(a_{1 i}, a_{2 i}\right),\left(q_{1 i}, q_{2 i}\right),\left(b_{1 i}, b_{2 i}\right)\right)>$ 0 . Consider the sequence $\left\{q_{1}=q_{10}, q_{11}, \ldots, q_{1 n}=p_{1}\right\}$ and the sequence $\left\{a_{11}, a_{12}, \ldots, a_{1 n}\right\} \in X_{1}$ and $\left\{b_{11}, b_{12}, \ldots, b_{1 n}\right\} \in Y_{1}$ such that $\forall i=1, \ldots, n, \mu_{1}\left(q_{1 i-1}, a_{1 i}\right.$ $\left., q_{1 i}, b_{1 i}\right)>0$, hence $M_{1}$ is connected. Consider the sequence $\left\{q_{2}=q_{20}, q_{21}, \ldots, q_{2 n}=p_{2}\right\}$ and the sequence $\left\{a_{21}, a_{22}, \ldots, a_{2 n}\right\} \in X_{2}$ and $\left\{b_{21}, b_{22}, \ldots, b_{2 n}\right\} \in Y_{2}$ such that $\forall i=1, \ldots, n, \mu_{2}\left(q_{2 i-1}, a_{2 i}, q_{2 i}, b_{2 i}\right)>0$, hence $M_{2}$ is connected.

Theorem 3.12 Let $M_{i}=\left(Q_{i}, X_{i}, Y_{i}, \mu_{i}\right)$ be a fmms, $i=1,2$ If fmm $M_{1} \odot M_{2}$ is connected then $M_{1}$ and $M_{2}$ are connected, where $\odot$ is restricted direct product,cascade product and wreath product.

Proof. 1) Suppose $\odot$ is restricted direct product. Suppose $M_{1} \odot M_{2}$ is connected. Let $\left(q_{1}, q_{2}\right),\left(p_{1}, p_{2}\right) \in Q_{1} \times Q_{2}$, Now $\exists$ a sequence of states $\left\{\left(q_{10}, q_{20}\right),\left(q_{11}, q_{21}\right), \ldots,\left(q_{1 n}, q_{2 n}\right)\right\} \in$ $Q_{1} \times Q_{2}$ and the sequence $\left\{a_{1}, a_{2}, \ldots, a_{n}\right\} \in X$ and $\left\{b_{1}, b_{2}, \ldots, b_{n}\right\} \in Y \forall i=1,2, \ldots, n$ either $\mu_{1} \odot \mu_{2}\left(\left(q_{1 i-1}, q_{2 i-1}\right), a_{i},\left(q_{1 i}, q_{2 i}\right), b_{i}\right)>0 \quad$ or $\mu_{1} \odot \mu_{2}\left(\left(q_{1 i}, q_{2 i}\right), a_{i},\left(q_{1 i-1}, q_{2 i-1}\right), b_{i}\right)>0$. Without loss of generality, suppose $\mu_{1} \odot \mu_{2}\left(\left(q_{1 i-1}, q_{2 i-1}\right), a_{i},\left(q_{1 i}, q_{2 i}\right), b_{i}\right)>$ 0 . Consider the sequence $\left\{q_{1}=q_{10}, q_{11}, \ldots, q_{1 n}=p_{1}\right\}$ and the sequence $\left\{a_{1}, a_{2}, \ldots, a_{n}\right\} \in X$ and $\left\{b_{1}, b_{2}, \ldots, b_{n}\right\} \in Y$ such that $\forall i=1, \ldots, n, \mu_{1}\left(q_{1 i-1}, a_{i}, q_{1 i}, b_{i}\right)>0$ and consider the sequence $\left\{q_{2}=q_{20}, q_{21}, \ldots, q_{2 n}=p_{2}\right\}$ and the sequence $\left\{a_{1}, a_{2}, \ldots, a_{n}\right\} \in X$ and $\left\{b_{1}, b_{2}, \ldots, b_{n}\right\} \in Y$ such that $\forall i=1, \ldots, n, \mu_{2}\left(q_{2 i-1}, a_{i}, q_{2 i}, b_{i}\right)>0$.

2) Suppose $\odot$ is cascade product. Suppose $M_{1} \odot M_{2}$ is connected. Let $\left(q_{1}, q_{2}\right),\left(p_{1}, p_{2}\right) \in Q_{1} \times Q_{2}$, Now $\exists$ a sequence of states $\left\{\left(q_{10}, q_{20}\right),\left(q_{11}, q_{21}\right), \ldots,\left(q_{1 n}, q_{2 n}\right)\right\} \in$ $Q_{1} \times Q_{2}$ and the sequence $\left\{x_{21}, x_{22}, \ldots, x_{2 n}\right\} \in X_{2}$ and $\left\{y_{21}, y_{22}, \ldots, y_{2 n}\right\} \in Y_{2} \forall i=1,2, \ldots, n$ either $\mu_{1} \odot \mu_{2}\left(\left(q_{1 i-1}, q_{2 i-1}\right), x_{2 i},\left(q_{1 i}, q_{2 i}\right), y_{2 i}\right)>0$ or $\mu_{1} \odot$ $\mu_{2}\left(\left(q_{1 i}, q_{2 i}\right), x_{2 i},\left(q_{1 i-1}, q_{2 i-1}\right), y_{2 i}\right)>0$. Without loss of generality, suppose $\mu_{1} \odot \mu_{2}\left(\left(q_{1 i-1}, q_{2 i-1}\right), x_{2 i},\left(q_{1 i}, q_{2 i}\right), y_{2 i}\right)>0$. Consider the sequence $\left\{q_{1}=q_{10}, q_{11}, \ldots, q_{1 n}=p_{1}\right\}$ and the sequence $\left\{\omega_{x}\left(q_{21}\right)=x_{11}\right.$ (say), $\omega_{x}\left(q_{22}\right)=$ $\left.x_{12}, \ldots, \omega_{x}\left(q_{2 n}\right)=x_{1 n}\right\} \in X_{1}$ and $\left\{\omega_{y}\left(q_{21}\right)\right.$ $\left.=y_{11}, \omega_{y}\left(q_{22}\right)=y_{12}, \ldots, \omega_{y}\left(q_{2 n}\right)=y_{1 n}\right\} \in Y_{1}$ such that $\forall i=1, \ldots, n, \mu_{1}\left(q_{1 i-1}, x_{1 i}, q_{1 i}, y_{1 i}\right)>0$. Now consider the sequence $\left\{q_{2}=q_{20}, q_{21}, \ldots, q_{2 n}=p_{2}\right\}$ and the sequence $\left\{x_{21}, x_{22}, \ldots, x_{2 n}\right\} \in X_{2}$ and $\left\{y_{21}, y_{22}, \ldots, y_{2 n}\right\} \in Y_{2}$ such that $\forall i=1, \ldots, n, \mu_{2}\left(q_{2 i-1}, x_{2 i}, q_{2 i}, y_{2 i}\right)>0$.

3) Suppose $\odot$ is wreath product. Suppose $M_{1} \odot M_{2}$ is connected. Let $\left(q_{1}, q_{2}\right),\left(p_{1}, p_{2}\right) \in Q_{1} \times Q_{2}$, Now $\exists$ a sequence of states $\left\{\left(q_{10}, q_{20}\right),\left(q_{11}, q_{21}\right), \ldots,\left(q_{1 n}, q_{2 n}\right)\right\} \in Q_{1} \times Q_{2}$ and the sequence $\left\{\left(f_{1}, x_{21}\right),\left(f_{2}, x_{22}\right), \ldots,\left(f_{n}, x_{2 n}\right)\right\} \in$ $X_{1}^{Q_{2}} \times X_{2}$ and $\left\{\left(g_{1}, y_{21}\right),\left(g_{2}, y_{22}\right), \ldots,\left(g_{n}, y_{2 n}\right)\right\} \in$ $Y_{1}^{Q_{2}} \times Y_{2}, \quad \forall i=1,2, \ldots, n$ either $\mu_{1} \odot$ $\mu_{2}\left(\left(q_{1 i-1}, q_{2 i-1}\right),\left(f_{i}, x_{2 i}\right),\left(q_{1 i}, q_{2 i}\right),\left(g_{i}, y_{2 i}\right)\right)$

$0 \quad$ or $\mu_{1} \odot \mu_{2}\left(\left(q_{1 i}, q_{2 i}\right),\left(f_{i}, x_{2 i}\right),\left(q_{1 i-1}, q_{2 i-1}\right)\right.$, $\left.\left(g_{i}, y_{2 i}\right)\right)>0$. Without loss of generality, suppose $\mu_{1} \odot \mu_{2}\left(\left(q_{1 i-1}, q_{2 i-1}\right),\left(f_{i}, x_{2 i}\right),\left(q_{1 i}, q_{2 i}\right),\left(g_{i}, y_{2 i}\right)\right)>0$. Consider the sequence $\left\{q_{1}=q_{10}, q_{11}, \ldots, q_{1 n}=p_{1}\right\}$ and the sequence $\left\{f_{1}\left(q_{21}\right)=x_{11}(\right.$ say $), f_{2}\left(q_{22}\right)=$ $\left.x_{12}, \ldots, f_{n}\left(q_{2 n}\right)=x_{1 n}\right\} \in X_{1}$ and $\left\{g_{1}\left(q_{21}\right)=\right.$ $y_{11}$ (say), $\left(g_{2}\left(q_{22}\right)=y_{12}, \ldots, g_{n}\left(q_{2 n}\right)=y_{1 n}\right\} \in Y_{1}$ such that $\forall i=1, \ldots, n, \mu_{1}\left(q_{1 i-1}, x_{1 i}, q_{1 i}, y_{1 i}\right)>0$. Now consider the sequence $\left\{q_{2}=q_{20}, q_{21}, \ldots, q_{2 n}=p_{2}\right\}$ and the sequence $\left\{x_{21}, x_{22}, \ldots, x_{2 n}\right\} \in X_{2}$ and $\left\{y_{21}, y_{22}, \ldots, y_{2 n}\right\} \in Y_{2}$ such that $\forall i=1, \ldots, n, \mu_{2}\left(q_{2 i-1}, x_{2 i}, q_{2 i}, y_{2 i}\right)>0$.

The converse of the above theorem is true when individual fmms are strongly connected.
Theorem 3.13 Let $M_{i}=\left(Q_{i}, X_{i}, Y_{i}, \mu_{i}\right)$ be a strongly connected fmms, $i=1,2$. Then $M_{1} \odot M_{2}$ is connected, where $\odot$ is restricted direct product, cascade product and wreath product.

Remark 3.4 Let $M_{i}=\left(Q_{i}, X_{i}, Y_{i}, \mu_{i}\right)$ be a fmms. Then

(1) by Theorem (2.13),(3.8) to (3.13) fmm $M_{1} \odot M_{2}$ is strongly connected if and only if $M_{1}$ and $M_{2}$ are strongly connected , where $\odot$ is cartesian product, full direct product restricted direct product, cascade product and wreath product.

(2) by Theorem (2.4) the topology induced by $M_{1} \odot M_{2}$ is discrete if and only if the topologies induced by $M_{1}$ and $M_{2}$ are discrete.

\section{CONCLUSION}

This paper is the study of fuzzy Mealy machines via topology, $\tau$, defined by the successor function on their set of states. For this purpose, various kinds of fuzzy Mealy machines such as cyclic, retrievable, strongly connected and connected are introduced. These kinds of fuzzy Mealy machines are discussed with the help of this topology. The main findings of this paper are:

(i) The fuzzy Mealy machine $M$ is strongly connected if and only if $\tau$ is the discrete topology on state set of M.

(ii)The fuzzy Mealy machine $M$ is connected if and only if it has no separated submachine.

(iii) The cartesian product ( full direct product) of two fuzzy Mealy machines is cyclic ( respectively retrievable, union of strongly connected submachines and connected) if and only if they are individually cyclic ( respectively retrievable, union of strongly connected submachines and connected).

(iv) If the restricted direct (cascade, wreath) product two fuzzy Mealy machines is cyclic ( respectively retrievable, union of strongly connected submachines, connected) then they are individually cyclic ( respectively retrievable, union of strongly connected submachines, connected). The converse of this result is true if both the fuzzy Mealy machines are strongly connected. This paper will definitely leads to a study of decomposition as well as minimization of fuzzy Mealy machines from different angle, which may be the direction of further research. Moreover, one can introduce and study category of fuzzy Mealy machines. Also, various concepts from category theory as well as from fuzzy Mealy machines can be verified using the topology introduced in this paper.

This paper is concluded with the following open problem for further study. What kind of topologies are generated by the products of fuzzy Mealy machines? In our opinion these topologies will be very much different from so far known topologies. However, one can guess the nature of these topologies with the help of the theorems proved in this paper (see remark 3.4(2) and definition 2.8)

\section{REFERENCES}

[1] Chaudhari S.R. and Desai A. S., On fuzzy Mealy and Moore machines, Bulletin of Pure and Applied Matheamtics, Vol.4(2), pp.375-384,2010.

[2] Chaudhari S. R. and Morye S. A., "Mealy-type finite state machines: A Fuzzy Approach", International J. of Math. Sci. and Engg. Appls., Vol.4(II), pp. 407-426, June 2010.

[3] Y. Li, and W.Pedrycz, Mo, "The equivalence between fuzzy Mealy and fuzzy Moore machines", Soft Computing, Vol.10(10) pp. 953-959, 2006.

[4] Jun Liu, Zhiwen Mo, Dong Qiu, Yang Wang, "Products of Mealy-type fuzzy finite state machines", Fuzzy Sets and Systems, Vol.160(16), pp. 2401-2415, 2010.

[5] Kendel A. and Lee S. C., Fuzzy Switching and Automata, Theory and applications, Crane, Russak, 1980 
[6] Kumbhojkar H.V and Chaudhari S.R., On covering of products of fuzzy finite state machines, Int. J. of Fuzzy Sets and Systems ,Vol.125 (2002), pp. 215-222.

[7] Li Y. M. and Pedryez W., "Minimization of lattice finite automata and its application to decomposition of lattice language", Fuzzy Sets and Systems, Vol.158(13), pp.14231436, 2007.

[8] Malik D. S. , Mordeson J. N. , and Sen M. K. , Submachines of fuzzy finite state machine, Journal of Fuzzy Mathematics, Vol. 2, pp. 781-92, 1994.

[9] Malik D. S., Mordeson J. N., and Sen M. K., The Cartesian composition of fuzzy finite state machines, Kybernetics, Vol 24 No.4,pp. 98-110, 1995.
[10] Mordeson J. N. and Malik D. S., Fuzzy Automata and Language : Theory and Applications, Boca Raton London, Chapman Hall/CRC, 2002.

[11] Mordeson J. N. and Nair P. S. ,Fuzzy Mealy machines : homomorphism, admissible relations and minimal machines, International Journal of Uncertainty, Fuzziand Knowledge-Based Systems, Vol 4 No.1,pp. 27-43, 1996.

[12] Shrivastava A. K. and Tiwari S.P., "On another decomposition of fuzzy automata", Journal of Uncertain Systems, Vol.5, No.1, pp.33-37, 2011.

[13] Wee W. G. and Fu K. S., A formulation of fuzzy automata and its application as a model of learning systems.IEEE Trans. SMC Vol.5(3), pp.215-223,1969. 